\title{
Accurate multi-level schemes for advection
}

\author{
Cheolwan $\mathrm{Kim}^{*, \dagger}$ \\ W. M. Keck Foundation Lab for Computational Fluid Dynamics, Department of Aerospace Engineering, \\ The University of Michigan, Ann Arbor, MI 48109-2140, U.S.A.
}

\begin{abstract}
SUMMARY
The upwind leapfrog method for the advection equation, which is non-dissipative and very accurate, is extended to higher-order and multiple dimensions. The higher-order version is developed by extending the stencil into space and time, and an analysis of the phase error is given. The schemes are then successfully applied to the classical test cases of rotating flow, and to a more realistic problem of non-uniform advection. Copyright (c) 2003 John Wiley \& Sons, Ltd.
\end{abstract}

KEY WORDS: leapfrog; upwind; advection; time reversible; non-dissipative

\section{INTRODUCTION}

Accurate numerical solution of the scalar advection equation is required in many contexts but designing such numerical procedure is very difficult, because of the well-known tendency of formally accurate methods to produce overshoots in regions where the data vary rapidly.

Accuracy by itself can be sought by several approaches. The simplest is to adopt increasingly high-order interpolation, such as spectral analysis. This tends to produce very broad numerical stencils, especially if the temporal accuracy is obtained, as it often is, from higherorder time integration method such as Runge-Kutta. Another way to increase the data available for interpolation is to include information from more than one time-step. The simplest example is the leapfrog method, which is naturally non-dissipative and energy preserving. Leapfrog methods, however, have a poor reputation among practitioners because spurious oscillations are often pronounced, and the method often responds unstably to boundary conditions, mesh irregularity, or non-linear terms.

A variant of the leapfrog method was proposed by Iserles [1] for the one-dimensional (1D) advection equation, using stencils having only point symmetry and therefore the capability of reflecting an upwind bias. These have theoretical advantages over regular leapfrog methods, having much reduced phase error, more compact stencils and only positive group velocities. Therefore, the 1D equation has been extended to multi-dimensional system of equations

\footnotetext{
* Correspondence to: Cheolwan Kim, W. M. Keck Foundation Lab for Computational Fluid Dynamics, Department of Aerospace Engineering, The University of Michigan, Ann Arbor, MI 48109-2140, U.S.A.

† E-mail: cheolwan_kim@hotmail.com
}

Copyright (c) 2003 John Wiley \& Sons, Ltd.

Received 20 April 2000 Revised 9 September 2002 
(acoustics, Maxwell's equations, elastodynamics) by P.L. Roe [2]. In the present research, we will focus on multi-dimensional advection; particularly the $2 \mathrm{D}$ advection problem.

In Section 2, we briefly review the ideas of Iserles concerning three-level schemes, together with alternative methods of introducing additional information, such as the four-level and Hermitian schemes. The resolving power of these methods is remarkable, particularly those having fourth-order accuracy. The fourth-order three-level scheme yields less than $1 \%$ error with only four grid points per wavelength and less than $0.1 \%$ with six points. Both four-level upwind leapfrog and Hermitian schemes yield less than $1 \%$ error with only three grid points per wavelength and less than $0.3 \%$ with five points. Therefore, these higher-order schemes are able to update the solution precisely for long integration times.

In Section 3, we discuss the strategies for extending these advection schemes to higher dimensions. The extension is not unique, but we follow heuristic principles of maintaining symmetry and minimizing the stencil. Both three-level and four-level methods are treated. Numerical tests for some standard problems are presented in Section 3.4. Specifically, we show advection in various fixed directions, and also in a circular path, of an initial Gaussian 'hump' on a rather coarse grid. Even after six complete revolutions the amplitude is almost perfectly preserved and the oscillations are negligable. Finally, we demonstrate an application on a polar grid with a 'realistic' velocity field, defined by creeping flow around a circular cylinder.

While these methods are simple, explicit and fully discrete, they are computationally expensive in storage because of the need to store more than one time level of data. The quality of the results obtained, however, is remarkable enough to support the use of these methods by practitioners.

\section{ONE-DIMENSIONAL ADVECTION}

The 1D advection equation for an unknown scalar $u(x, t)$, applied to an initial-value problem, $\partial u / \partial t+a \cdot \partial u / \partial x=0$, advects the given profile at the speed, $a$. Before any detailed analysis of the upwind leapfrog scheme, a few classical techniques are compared through simple numerical experiment. The left boundary of the computational domain is excited by a sinusoidal function which is propagated to the right-hand side at the speed, $a$ without any dissipation. Figure 1 compares four different numerical test results with eight grid points per wavelength $(N=8)$. Figure 1(a) presents the result of the second-order Lax-Wendroff scheme. After travelling only one wavelength (ten iterations), it begins to show some deviation from the analytic solution (dotted line) and produces substantial dispersion and dissipation errors after travelling ten wavelengths (200 iterations). Figure 1(b) is updated by the simple upwind scheme which has a huge dissipation error and shows that most of the amplitude is dissipated after travelling three wavelengths. Neither of these schemes is suitable for accurately simulating advection, and would require many more grid points to get a reasonable result.

The third picture, Figure 1(c), is obtained with the regular leapfrog scheme, which uses three levels and is time-reversible (Figure 3). Therefore, it has no dissipation and maintains the original amplitude. However, it has a large dispersion error and still requires a lot more grid to get reasonable phase resolution. The last picture (Figure 1(d)) shows the result of the upwind leapfrog scheme proposed by Iserles [1]. It is again time-reversible and keeps the original wave amplitude without any dissipation. Furthermore the biased stencil maintains the 

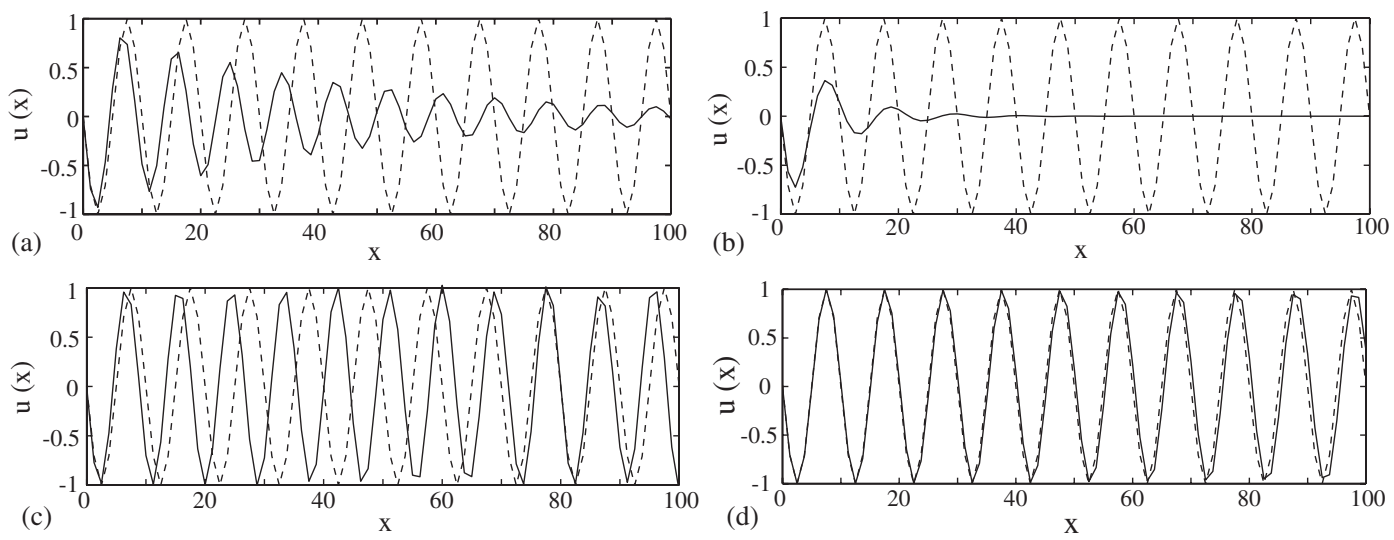

Figure 1. Numerical results of four different numerical schemes. Solid line: Numerical solution. Dotted line: Analytic solution. Courant number, $v=0.4$. Grid points per wavelength, $N=8$. (a) Lax-Wendroff scheme; (b) simple upwind scheme; (c) regular leapfrog scheme; and (d) upwind leapfrog scheme
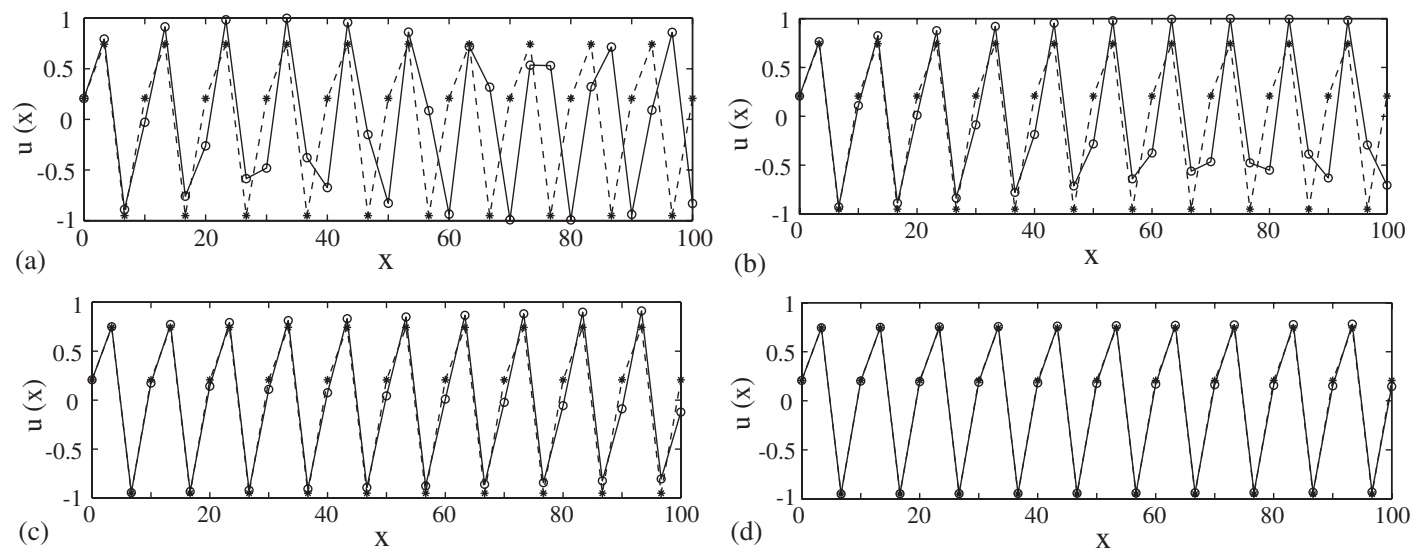

Figure 2. Numerical results of four upwind leapfrog schemes. Solid line and circle: Numerical solution. Dotted line and star: Analytic solution. Courant number, $v(a),(b)=0.4,(c),(d)=0.2$. Grid Points per wavelength, $N=3$. (a) Second-order scheme; (b) space-extended fourth-order scheme; (c) time-extended fourth-order scheme; and (d) space and time-extended sixth-order scheme.

phase speed more accurately than the regular leapfrog scheme. This comparison confirms that the upwind leapfrog method has better accuracy than other second-order methods.

Another experiment, shown in Figure 2, demonstrated the accuracy of the second-, fourthand sixth-order upwind leapfrog methods on a coarse grid, $N=3$. Although the analytic solution, marked by star $(*)$ and dotted line, is a smooth sine wave, the interpolant of the analytic solution appears discrete. The result of the second-order scheme shown in Figure 2(a) begins to show a dispersion error after travelling one wavelength. Two fourth-order schemes developed by extending the second-order scheme in space and time, demonstrate the results 

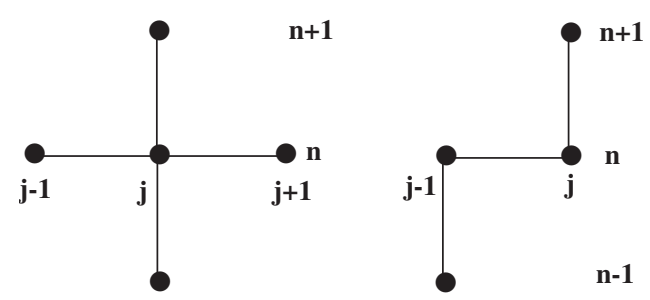

Figure 3. Stencils of leapfrog type schemes. Left: Regular leapfrog. Right: Upwind leapfrog.

in Figure 2(b) and 2(c). They preserve the phase precisely. However, to graphical resolution, the method using the time-extended stencil is more accurate than the one extended into space. An heuristic reason for it being better than the stencil extended in space is that it has a smaller extent in the characteristic co-ordinate $x$-at. The sixth-order scheme based on the stencil shown in Figure 5(c) preserves the phase with extraordinary accuracy for such an under-resolved grid. In what follows, we successfully extend both fourth-order methods to 2D grids; extension of the corresponding sixth-order version remains to be achieved.

\subsection{One-dimensional upwind leapfrog methods and analysis}

2.1.1. Second-order schemes. For positive wave speed, $a>0$, the regular leapfrog and upwind leapfrog schemes employ the stencils of Figure 3, and are time-reversible.

$$
\begin{aligned}
& \text { Regular leapfrog: } u_{j}^{n+1}=u_{j}^{n-1}-v\left(u_{j+1}^{n}-u_{j-1}^{n}\right) \\
& \text { Upwind leapfrog: } u_{j}^{n+1}=u_{j-1}^{n-1}+(1-2 v)\left(u_{j}^{n}-u_{j-1}^{n}\right)
\end{aligned}
$$

where $v=a \Delta t / \Delta x$.

For the analysis of two schemes, von Neumann analysis is performed by taking the discrete solution $u_{j}^{n}$ to be sampled from a continuous function

$$
u(x, t)=\mathrm{e}^{\mathrm{i}(\omega t-\kappa x)}
$$

where $\kappa$ is the given real spatial frequency of the data, and $\omega$ is the temporal frequency. Fourier angles $\theta$ and $\phi$ are introduced with the definitions of

$$
\kappa=\frac{\theta}{\Delta x}=\frac{2 \pi}{N \Delta x}, \quad \omega=\frac{\phi}{\Delta t}
$$

and the amplification factors are defined by $g=\mathrm{e}^{\mathrm{i} \omega \Delta t}$. For a reversible and stable scheme, $\omega$ is purely real and its exact value is $\omega=a \kappa$. The phase error is defined as

$$
E_{\mathrm{p}}(\%)=\left(\frac{\omega}{a \kappa}-1\right) \times 100
$$

The results are presented as contour plots of equal phase error, on diagrams where the axes are $v(\geqslant 0)$ and $N(\geqslant 2)$. The results for the regular and upwind leapfrog schemes are shown in Figure 4. 


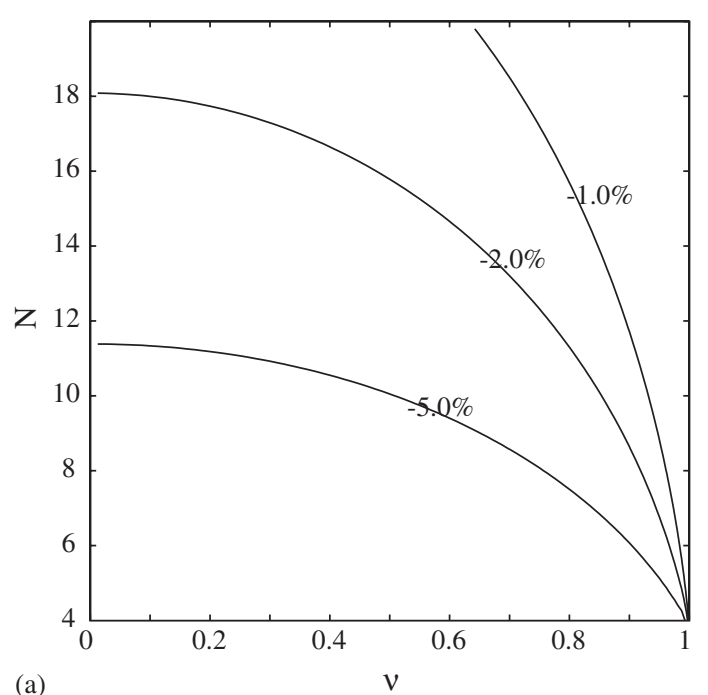

(a)

Figure 4. Phase properties of second-order leapfrog schemes: (a) regular

Figure 4. Phase properties of second-order leapfrog schemes: (a) regular
leapfrog; and (b) upwind leapfrog.

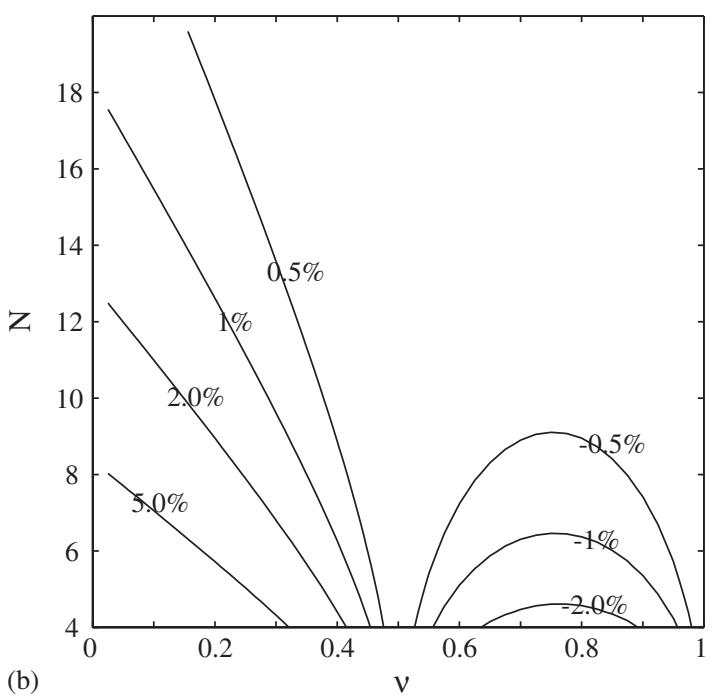

(b)

Note that a scheme having $\mathrm{K}$ time levels leads to a polynomial of order $K-1$ in $g$ and hence to $K-2$ spurious solutions that do not approximate the differential problem. At this point we ignore such roots, although in higher dimensions they will become an issue. The time-reversible schemes are unstable whenever any root $g$ lies outside the unit circle, and we only plot those cases for which all roots are stable.

Note also that the upwind leapfrog scheme has vanishing error when $v=\frac{1}{2}$, which explains why the error is small over the entire range $0 \leqslant v \leqslant 1.0 .^{\ddagger}$

\subsection{Higher-order extensions of the upwind leapfrog scheme}

To get higher-order accuracy, more information is necessary beyond that used in the secondorder upwind leapfrog scheme. To provide the information, the stencil is extended in space or time. The first is to extend the stencil of the second-order scheme in space, as shown in Figure 5(a) but this makes the scheme less compact and is difficult to apply at the boundary. The second is to stretch the stencil in time by including data from time level $n-2$ (Figure 5(b)). This results in a very accurate scheme that is exact both when $v=\frac{1}{2}$ and when $v=\frac{1}{3}$. However, this scheme is unstable for $v>\frac{1}{2}$. Another method is to combine these methods. Figure 5(c) shows the stencil of the sixth-order scheme obtained by extending the second-order stencil in space and time.

\footnotetext{
¥ Prof. P. M. Gresho has pointed out that the upwind leapfrog scheme, Equation (2), reduces for very small timesteps to the semi-discrete 'box scheme', $\frac{1}{2}\left(\dot{u}_{j}+\dot{u}_{j-1}\right) /(\Delta x)$ which does not have a particularly accurate phase speed, because in this limit the benefits of upwinding are lost. In fact, all of the schemes discussed here are improved by being run as fast as possible, which is typical of good fully discrete schemes.
} 


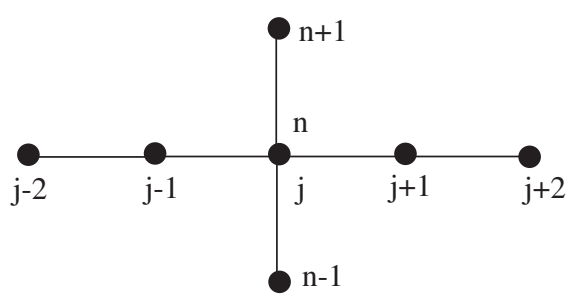

(a)

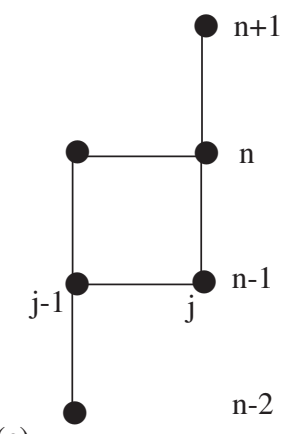

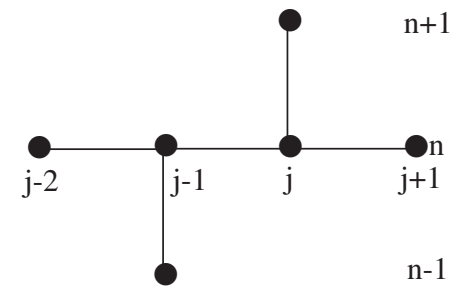

(b)

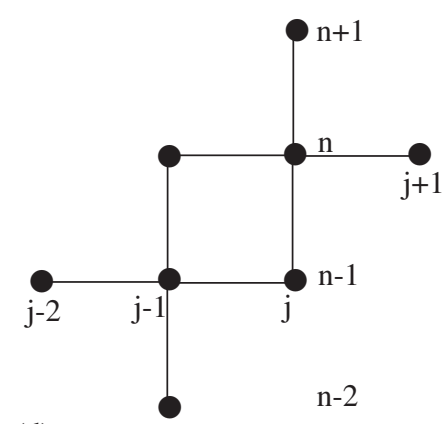

(d)

Figure 5. Stencils of the fourth- and sixth-order regular, (a) and upwind (b,c,d) Leapfrog schemes. (a) Fourth-order, (b) space-extended fourth-order, (c) time-extended fourth-order, (c) space- and time-extended sixth-order.

The scheme with the stencil extended in space, shown in Figure 5(b), is

$$
\begin{aligned}
u_{j}^{n+1}= & u_{j-1}^{n-1}+\frac{(v+1)(v-2)(2 v-1)}{2}\left(u_{j}^{n}-u_{j-1}^{n}\right) \\
& -\frac{v(v-1)(2 v-1)}{6}\left(u_{j+1}^{n}-u_{j-2}^{n}\right)
\end{aligned}
$$

and the scheme extended in time (Figure 5(c)) is

$$
\begin{aligned}
u_{j}^{n+1}= & u_{j-1}^{n-2}+2(1-3 v)\left(u_{j}^{n}-u_{j-1}^{n-1}\right) \\
& +\frac{(1-2 v)(1-3 v)}{v+1}\left(u_{j-1}^{n}-u_{j}^{n-1}\right)
\end{aligned}
$$

The sixth-order scheme whose stencil extended in space and time as shown in Figure 5(d) is

$$
\begin{aligned}
u_{j}^{n+1}= & u_{j-1}^{n-2}-4 \frac{(3 v-1)\left(v^{2}-1\right)}{v-2}\left(u_{j}^{n}-u_{j-1}^{n-1}\right) \\
& -\left(6 v^{2}-5 v+1\right)\left(u_{j}^{n-1}-u_{j-1}^{n}\right)+\frac{v^{2}\left(6 v^{2}-5 v+1\right)}{v^{2}-5 v+6}\left(u_{j+1}^{n}-u_{j-2}^{n-1}\right)
\end{aligned}
$$


Figure 6 presents the phase speed error, $E(\%)$ for the fourth- and sixth-order schemes. First of all, the space-extended fourth-order regular leapfrog scheme is presented in Figures 5(a) and 6(a). The fourth-order scheme demonstrates much higher accuracy than the second-order one but it still contains much more phase error than any fourth-order upwind leapfrog scheme, which justifies again the necessity of the upwind technique. As can be seen from Figures 4 and 6, the higher-order schemes produce much better accuracy than the second-order ones. The space-extended scheme has less accurate phase speed than the time-extended ones. However, its stability condition is $0 \leqslant v \leqslant 1$ and its memory requirement is the least since it is threelevel scheme. Its straightforward extension to multi-dimensional wave propagation problems is also possible [3]. The fourth-order scheme extended in time shows very accurate phase speed property. With only 3.7 grid points per wavelength, $1 \%$ error of phase speed accuracy can be maintained. Its compactness makes it easy to apply the scheme at the computational boundaries but its maximum Courant number is $v=\frac{1}{2}$. The sixth-order scheme shows very accurate phase properties and maintains the phase error less than $1 \%$ with only 2.6 grids per wavelength. Its maximum Courant number is also $v=\frac{1}{2}$. Neither of these schemes has yet been extended to multi-dimensional wave propagation. The easier extension to multi-dimensional advection is the topic of this paper.

\subsection{Non-constant wave speed}

If the advection speed is variable or the grid spacing is not uniform, a more careful approach is demanded to maintain the accuracy of the upwind leapfrog scheme [4]. The governing equation of this case can be simply written as

$$
\frac{\partial u}{\partial t}+a(x) \frac{\partial u}{\partial x}=0
$$

The modified equation of the second-order upwind leapfrog scheme is written as follows:

$$
\begin{aligned}
& \frac{u_{j}^{n+1}-u_{j}^{n}+u_{j-1}^{n}-u_{j-1}^{n-1}}{2 \Delta t}+\frac{a_{j}+a_{j-1}}{2} \frac{u_{j}^{n}-u_{j-1}^{n}}{\Delta x}=\frac{\partial u}{\partial t}+a \frac{\partial u}{\partial x} \\
& +\frac{\Delta x^{2}}{24}\left(a \frac{\partial^{3} u}{\partial x^{3}}+3 \frac{\partial^{3} u}{\partial x^{2} \partial t}+6 \frac{\Delta t}{\Delta x} \frac{\partial^{3} u}{\partial x \partial t^{2}}+4 \frac{\Delta t^{2}}{\Delta x^{2}} \frac{\partial^{3} u}{\partial t^{3}}\right)+O\left(\Delta x^{4}\right)
\end{aligned}
$$

With the relation, $\partial u / \partial t=-a(x) \partial u / \partial x$, the second-order truncation error terms of Equation (10) can be written as

$$
\frac{\Delta x^{2}}{24}\left[a \frac{\partial^{3} u}{\partial x^{3}}-3 \frac{\partial^{2}}{\partial x^{2}}\left(a \frac{\partial u}{\partial x}\right)+\left(6 \frac{\Delta t}{\Delta x}-4 a \frac{\Delta t^{2}}{\Delta x^{2}}\right) \frac{\partial}{\partial x}\left(a \frac{\partial}{\partial x}\left(a \frac{\partial u}{\partial x}\right)\right)\right]
$$

To maintain the fourth-order accuracy, the above terms should be discretized and subtracted from the second-order scheme. The method that was found to work best is based on writing (11) so that the correction can be added in conservation form as

$$
\frac{1}{24 \Delta t}\left[v_{j-1 / 2} \delta_{x}^{3} u_{j-1 / 2}^{n}-3 \delta_{x}^{2}\left(v_{j-1 / 2} \delta_{x} u_{j-1 / 2}^{n}\right)+\left(6-4 v_{j-1 / 2}\right) \delta_{x}\left[v_{j-1 / 2} \delta_{x}\left(v_{j-1 / 2} \delta_{x} u_{j-1 / 2}^{n}\right)\right]\right]
$$



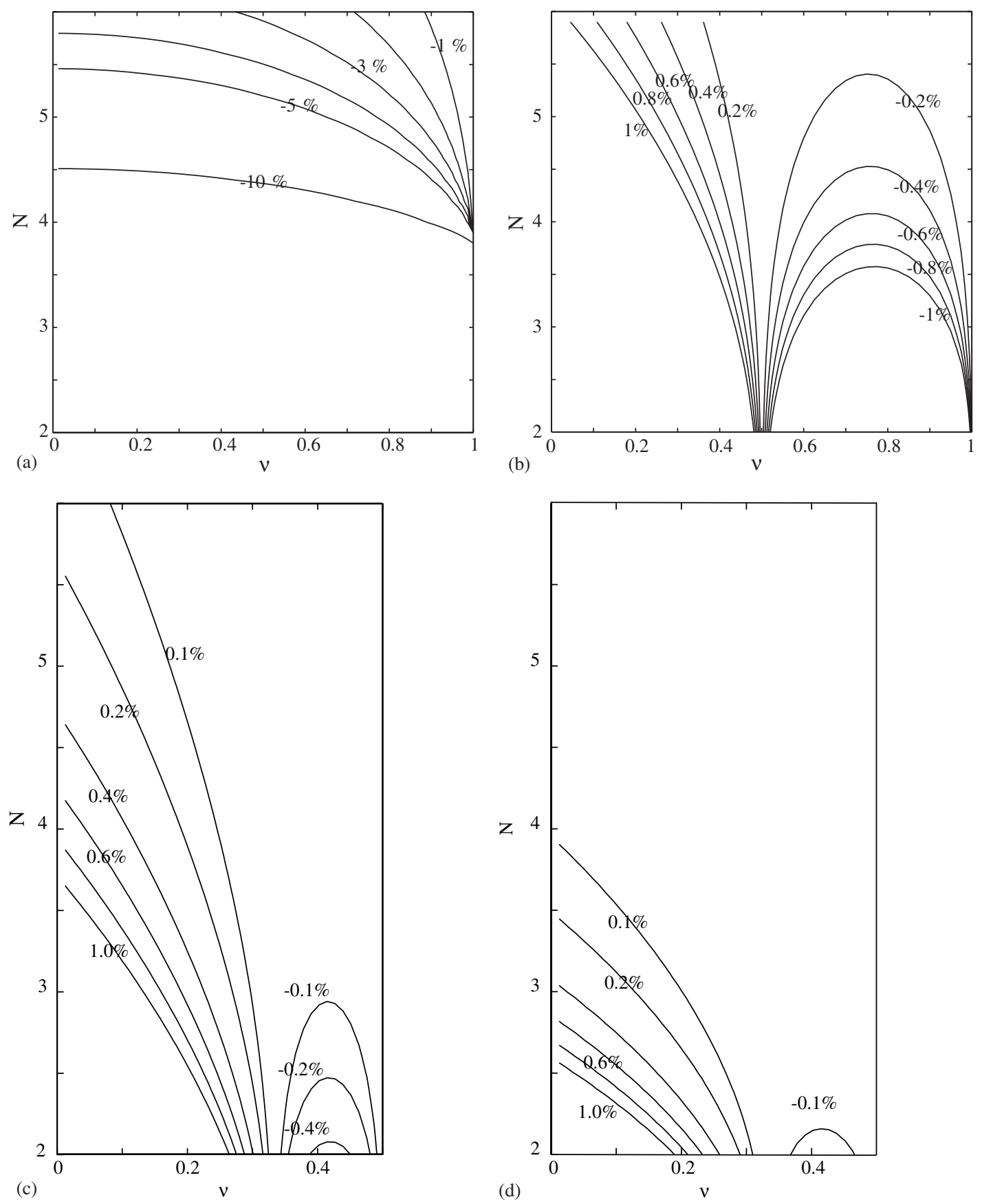

Figure 6. Phase error comparison of fourth- and sixth-order regular, (a) and upwind (b,c,d) leapfrog schemes. (a) Fourth-order, (b) space-extended fourth-order, (b) time-extended fourth-order, (c) space- and time-extended sixth-order. 
where $v=a \Delta t / \Delta x$ and the discrete differencing and averaging operators are defined by

$$
\delta_{x} u_{j}^{n}=u_{j+1 / 2}^{n}-u_{j-1 / 2}^{n}, \quad \mu_{x} u_{j}^{n}=\frac{1}{2}\left(u_{j+1 / 2}^{n}+u_{j-1 / 2}^{n}\right)
$$

This discretization is performed on the stencil shown in Figure 5(a). However there is an equivalent way to discretize for a smooth advection speed; use (a). It is central difference to expand the writing (11) and discretize on the stencil (Figure 5(a)).

Figure 7 compares two discretizations, central and conservative differences. The conservative difference is completed by subtracting the discretization (12) from the second-order scheme. The advection speed is discontinuous around the origin; (a): $0.1(x \leqslant 0), 1.0(x>0)$ (b): $1.0(x \leqslant 0), 0.1(x>0)$ and its ratio is 10 . Both results are compared with the analytic solution and the conservative scheme is shown to work well (Figure 7).

\section{TWO-DIMENSIONAL ADVECTION PROBLEMS}

The $2 \mathrm{D}$ advection equation is

$$
\frac{\partial u}{\partial t}+a \frac{\partial u}{\partial x}+b \frac{\partial u}{\partial y}=0
$$

and the advection speed, $(a, b)$ is assumed to be constant. The propagation direction, $\alpha$, is defined as $\alpha=\tan ^{-1}(b / a)$. In this section, the strategy for the stencil arrangement is described, higher-order versions of upwind leapfrog method are developed and their numerical applications are explained.

\subsection{Second-order advection schemes}

There are a few approaches to extend the upwind leapfrog method to multi-dimensions. They primarily depend on the computational grid type as mentioned by Roe and Thomas [4,5]. Two different mesh arrangements appear in Figure 8. The first arrangement is a uniform grid which stores a variable at the cell node and the second is a staggered grid storing the variable at the cell edge. Although the staggered grid is merely a scaling and rotation of the uniform grid, they are generally treated distinctly because in wave propagation problems, it is sometimes advantageous to store different variables at different nodes of the staggered mesh. Of course, in a scalar problem, there is no real distinction, but we adopt a staggered grid to remain compatible with the treatment of systems. The total number of computational grid points for the staggered grid is about twice that of a uniform grid in 2D problems and three times in three-dimensions. The second-order stencils are shown in Figure 9 and the discretized equations are

$$
\begin{aligned}
& \text { (a): } \delta_{t} u_{j+1 / 2, k}^{n+1 / 2}+\delta_{t} u_{j-1 / 2, k}^{n-1 / 2}+2 v_{x} \delta_{x} u_{j, k}^{n}+2 v_{y} \delta_{y} u_{j, k}^{n}=0 \\
& \text { (b): } \delta_{t} u_{j, k+1 / 2}^{n+1 / 2}+\delta_{t} u_{j, k-1 / 2}^{n-1 / 2}+2 v_{x} \delta_{x} u_{j, k}^{n}+2 v_{y} \delta_{y} u_{j, k}^{n}=0 \\
& \text { (c): } \delta_{t} u_{j-1 / 2, k}^{n+1 / 2}+\delta_{t} u_{j+1 / 2, k}^{n-1 / 2}+2 v_{x} \delta_{x} u_{j, k}^{n}+2 v_{y} \delta_{y} u_{j, k}^{n}=0 \\
& \text { (d): } \delta_{t} u_{j, k-1 / 2}^{n+1 / 2}+\delta_{t} u_{j, k+1 / 2}^{n-1 / 2}+2 v_{x} \delta_{x} u_{j, k}^{n}+2 v_{y} \delta_{y} u_{j, k}^{n}=0
\end{aligned}
$$



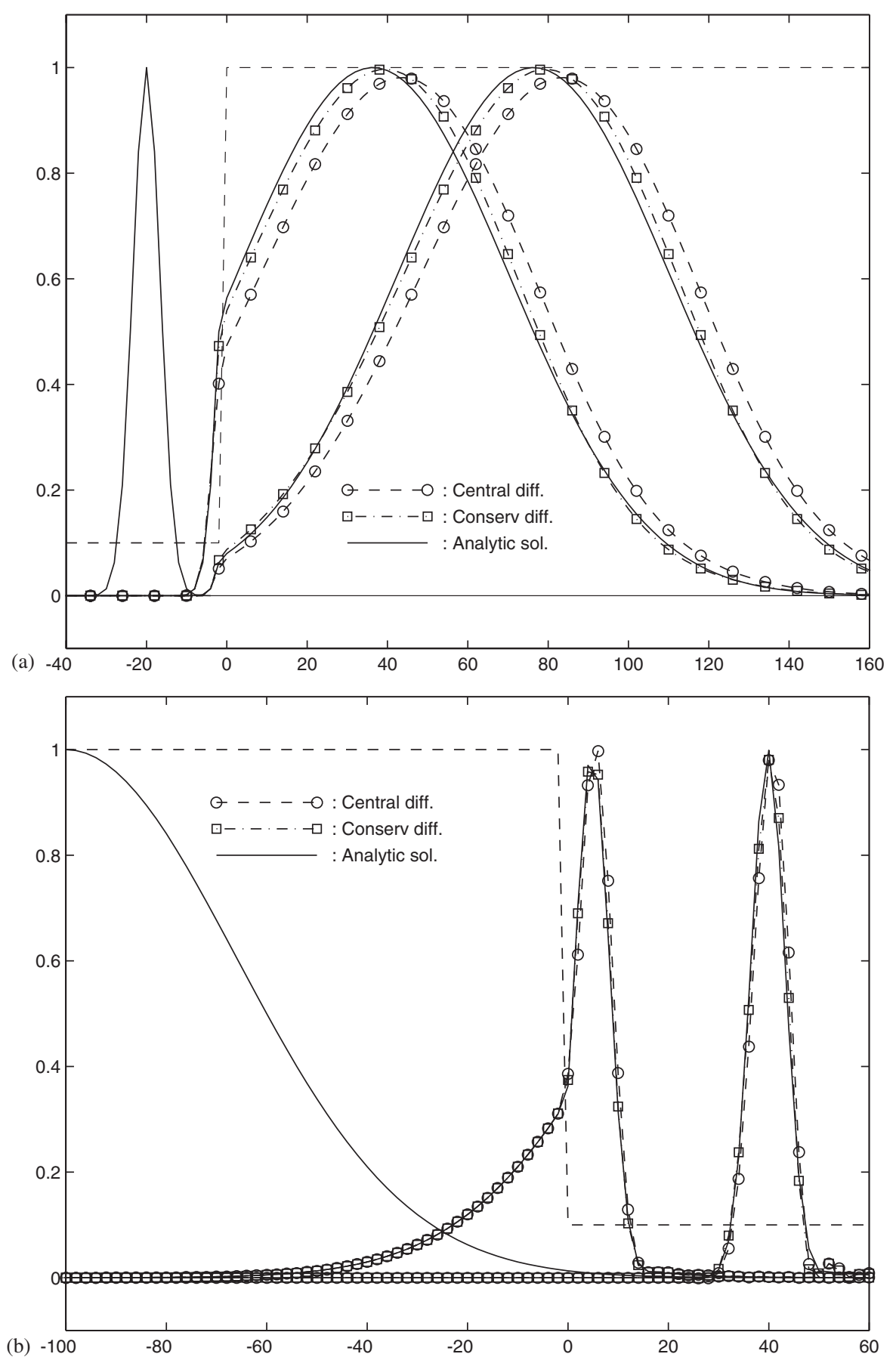

Figure 7. Comparison of two different discretizations for non-constant advection speed: (a) accelerating speed, from 0.1 to 1.0 ; (b) decelerating speed, from 1.0 to 0.1 . 


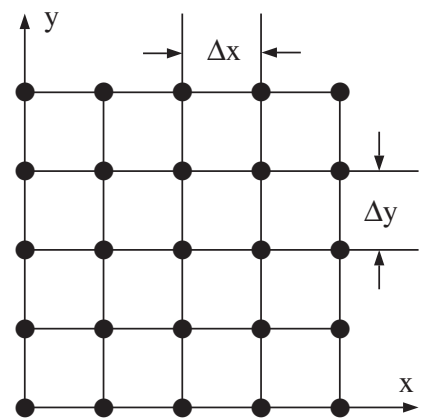

(a)

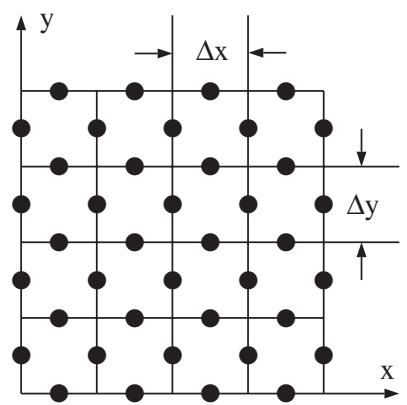

(b)

Figure 8. Grid arrangements: (a) uniform grid; (b) staggered grid.
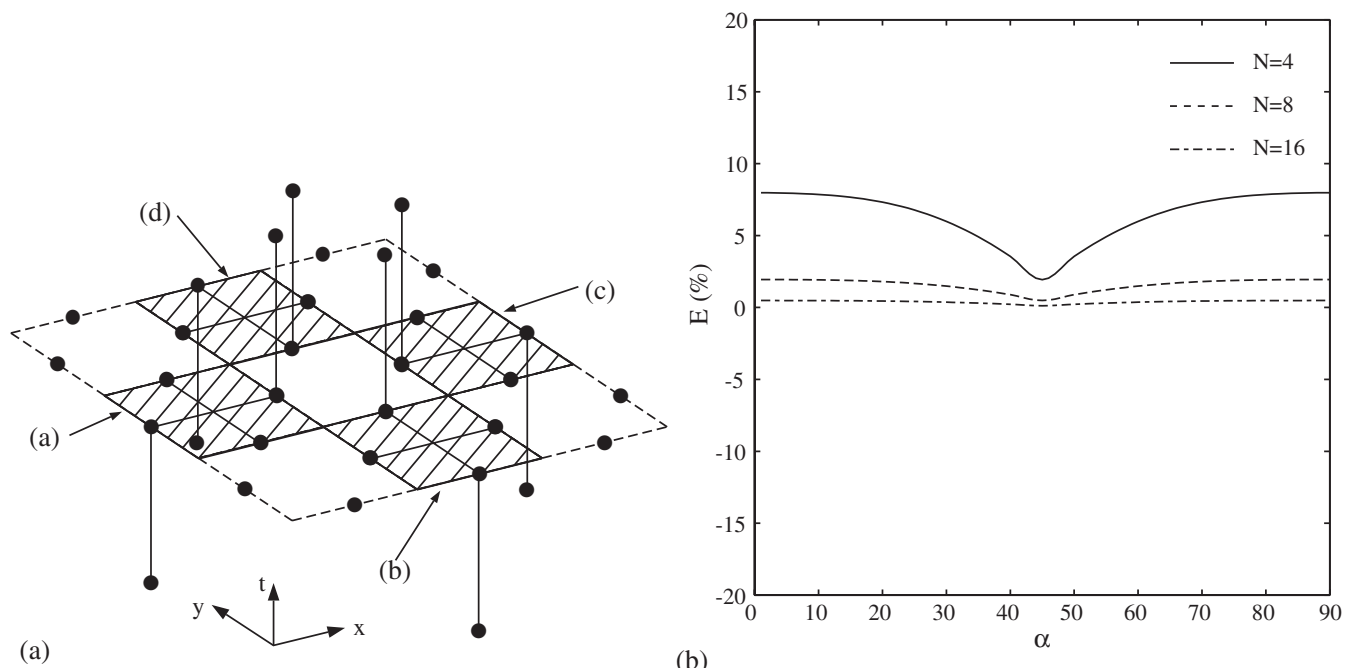

(b)

Figure 9. Stencils and phase error of second-order upwind leapfrog schemes.

where $v_{x}=a \Delta t / \Delta x, v_{y}=b \Delta t / \Delta y$ and the discrete differencing and averaging operators are defined by

$$
\begin{aligned}
& \delta_{t} u_{j, k}^{n}=u_{j, k}^{n+1 / 2}-u_{j, k}^{n-1 / 2}, \quad \mu_{t} u_{j, k}^{n}=\frac{1}{2}\left(u_{j, k}^{n+1 / 2}+u_{j, k}^{n-1 / 2}\right) \\
& \delta_{x} u_{j, k}^{n}=u_{j+1 / 2, k}^{n}-u_{j-1 / 2, k}^{n}, \quad \mu_{x} u_{j, k}^{n}=\frac{1}{2}\left(u_{j+1 / 2, k}^{n}+u_{j-1 / 2, k}^{n}\right) \\
& \delta_{y} u_{j, k}^{n}=u_{j, k+1 / 2}^{n}-u_{j, k-1 / 2}^{n}, \quad \mu_{y} u_{j, k}^{n}=\frac{1}{2}\left(u_{j, k+1 / 2}^{n}+u_{j, k-1 / 2}^{n}\right)
\end{aligned}
$$

We will adopt whichever of (a), (b), (c) or (d) respects the domain of dependence. This means that the solution may not depend continuously on the direction of propagation, and for computing steady-state solutions this might cause problems with convergence. However we have not found any difficulties due to this dependence in the linear scalar case. 


\subsection{Dispersion analysis}

A dispersion analysis is performed to investigate the phase properties of the $2 \mathrm{D}$ scheme. The solution is assumed to be

$$
u(x, y, t)=\exp \left[\mathrm{i}\left(\omega t-k_{x} x-k_{y} y\right)\right]=\exp \left[\mathrm{i}\left(n \phi-j \theta_{x}-k \theta_{y}\right)\right]
$$

where $\phi=\omega \Delta t, \theta_{x}=k_{x} \Delta x$ and $\theta_{y}=k_{y} \Delta y$. The exact phase speed $\phi_{\mathrm{e}}$ for the advection equation (14) is

$$
\phi_{\mathrm{e}}=v_{x} \theta_{x}+v_{y} \theta_{y}
$$

The propagation direction, $\alpha$, varies from 0 to $90^{\circ}$ and the wave front angle $\left(=\tan ^{-1}\left[k_{y} / k_{x}\right]\right)$ is chosen to be coincident with $\alpha$. The Fourier angles $\theta_{x}$ and $\theta_{y}$ are related to the number of cells-per-wavelength, $N$ and the wave front direction, $\alpha$ with the following relations:

$$
\theta_{x}=\frac{2 \pi}{N} \cos (\alpha), \quad \theta_{y}=\frac{2 \pi}{N} \sin (\alpha)
$$

The number of cells-per-wavelength is chosen as $N=4,8,16$ to examine the order of accuracy trends. The Courant numbers are chosen as

$$
v_{x}=\frac{1}{4} \cos (\alpha), \quad v_{y}=\frac{1}{4} \sin (\alpha)
$$

and the dispersion error, $E$ is defined as

$$
E(\%)=\left[\frac{\phi}{\phi_{\mathrm{e}}\left(=v_{x} \theta_{x}+v_{y} \theta_{y}\right)}-1\right] \times 100
$$

The errors of the upwind leapfrog scheme appear in Figure 9. The error is the least when the wave propagates oblique to the stencil and the blending function makes the phase speed change smooth around $45^{\circ}$.

\subsection{Higher-order upwind leapfrog schemes}

Although the second-order upwind leapfrog method is compact and has higher resolution than other second-order methods, its resolution is still not high enough to simulate the long range propagation problems. In this section, therefore, higher-order schemes are developed and examined. To increase the order of accuracy, the second-order stencil can be extended in either space or time.

3.3.1. Three-level space-extended fourth-order method. To increase the order of accuracy, the second-order stencil is extended in either space or time as was done in one dimension. The modified equation of the second-order upwind leapfrog method on the staggered grid is

$$
\begin{aligned}
& \delta_{t} u_{j+1 / 2, k}^{n+1 / 2}+\delta_{t} u_{j-1 / 2, k}^{n-1 / 2}+2\left(v_{x} \delta_{x}+v_{y} \delta_{y}\right) u_{j, k}^{n}=2 \Delta t\left(\frac{\partial u}{\partial t}+a \frac{\partial u}{\partial x}+b \frac{\partial u}{\partial y}\right) \\
& -c_{x x x} \Delta x^{3} \frac{\partial^{3} u}{\partial x^{3}}-c_{x x y} \Delta x^{2} \Delta y \frac{\partial^{3} u}{\partial x^{2} \partial y}-c_{x y y} \Delta x \Delta y^{2} \frac{\partial^{3} u}{\partial x \partial y^{2}}-c_{y y y} \Delta y^{3} \frac{\partial^{3} u}{\partial y^{3}}
\end{aligned}
$$



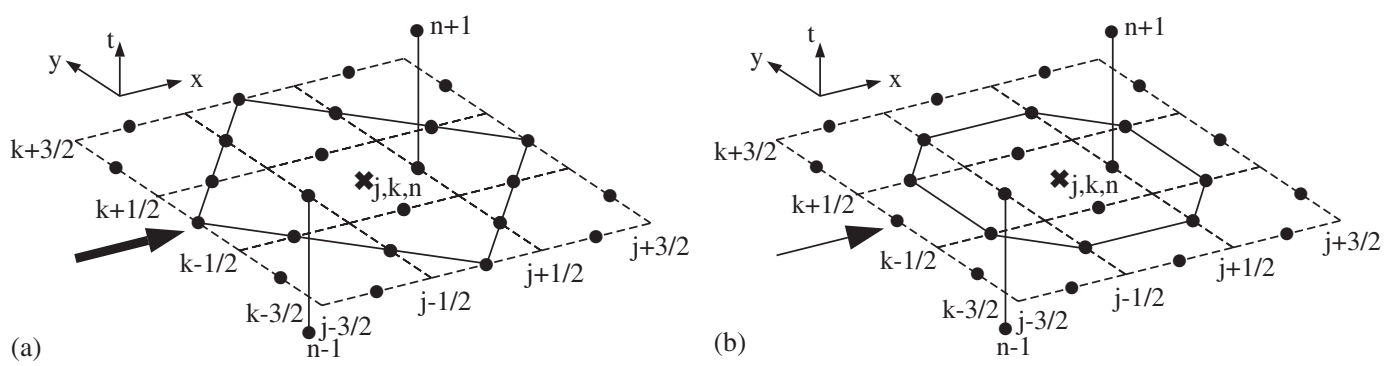

Figure 10. Space-extended three-level stencils for $x$-directional advection.

where

$$
\begin{aligned}
& c_{x x x}=\frac{1}{6} v_{x}\left(v_{x}-1\right)\left(2 v_{x}-1\right), \quad c_{x x y}=\frac{1}{4} v_{y}\left(2 v_{x}-1\right)^{2} \\
& c_{x y y}=\frac{1}{2} v_{y}^{2}\left(2 v_{x}-1\right), \quad c_{y y y}=\frac{1}{12} v_{y}\left(2 v_{y}-1\right)\left(2 v_{y}+1\right)
\end{aligned}
$$

for $-\pi / 4 \leqslant \alpha \leqslant \pi / 4$. To acquire the fourth-order accuracy, the truncation error terms are discretized on the stencil extended in space. The simplest replacements for the derivatives are

$$
\begin{aligned}
& \Delta x^{3} \frac{\partial^{3} u}{\partial x^{3}}=\delta_{x}^{3} u_{j, k}^{n}, \quad \Delta x^{2} \Delta y \frac{\partial^{3} u}{\partial x^{2} \partial y}=\delta_{x}^{2} \delta_{y} u_{j, k}^{n} \\
& \Delta y^{3} \frac{\partial^{3} u}{\partial y^{3}}=\delta_{y}^{3} u_{j, k}^{n}, \quad \Delta x \Delta y^{2} \frac{\partial^{3} u}{\partial x \partial y^{2}}=\delta_{x} \delta_{y}^{2} u_{j, k}^{n}
\end{aligned}
$$

as suggested by Thomas [5] but this leads to the rather large stencil of Figure 10(a). A more compact stencil, therefore, is investigated as shown in Figure 10(b). This stencil is again the one appropriate for a propagation direction aligned with $+x$, say $-\pi / 4 \leqslant \alpha \leqslant \pi / 4$. It is quite compact but still requires special treatment at boundaries. The finite difference equation based on Figure $10(b)$ is

$$
\begin{aligned}
& \delta_{t} u_{j+1 / 2, k}^{n+1 / 2}+\delta_{t} u_{j-1 / 2, k}^{n-1 / 2}+2\left(v_{x} \delta_{x}+v_{y} \delta_{y}\right) u_{j, k}^{n}+c_{x x x}\left(8-8 \mu_{x} \mu_{y}+\delta_{y}^{2}\right) \delta_{x} u_{j, k}^{n} \\
& \quad+c_{x x y} \delta_{x}^{2} \delta_{y} u_{j, k}^{n}+c_{x y y} \delta_{x} \delta_{y}^{2} u_{j, k}^{n}+c_{y y y}\left(8-8 \mu_{x} \mu_{y}+\delta_{x}^{2}\right) \delta_{y} u_{j, k}^{n}=0
\end{aligned}
$$

The set of difference equations for other propagation directions are presented in Appendix A.

3.3.2. Four-level time-extended fourth-order method. To develop schemes of fourth-order accuracy, the stencil of the second-order method is extended into time. The second-order truncation error terms of the upwind leapfrog scheme are presented as

$$
\begin{aligned}
& \delta_{t} u_{j+1 / 2, k}^{n+1}+\delta_{t} u_{j-1 / 2, k}^{n-1}+2\left(v_{x} \delta_{x}+v_{y} \delta_{y}\right) \mu_{t} u_{j, k}^{n}=2 \Delta t\left(\frac{\partial u}{\partial t}+a \frac{\partial u}{\partial x}+b \frac{\partial u}{\partial y}\right) \\
& +\frac{\Delta x^{2} \Delta t}{12}\left[a \frac{\partial^{3} u}{\partial x^{3}}+b \frac{\Delta y^{2}}{\Delta x^{2}} \frac{\partial^{3} u}{\partial y^{3}}+3 \frac{\partial^{3} u}{\partial x^{2} \partial t}+12 \frac{\Delta t}{\Delta x} \frac{\partial^{3} u}{\partial x \partial t^{2}}+10 \frac{\Delta t^{2}}{\Delta x^{2}} \frac{\partial^{3} u}{\partial t^{3}}\right]+O\left(\Delta x^{4}\right)
\end{aligned}
$$




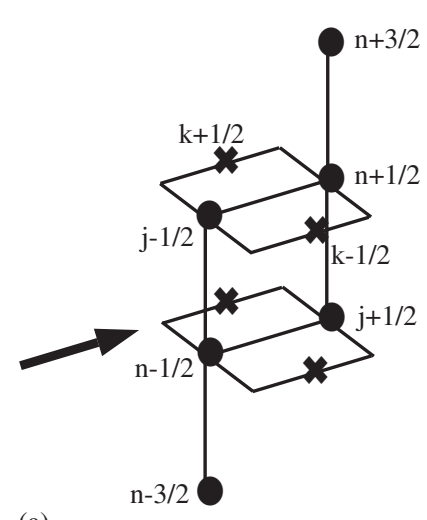

(a)

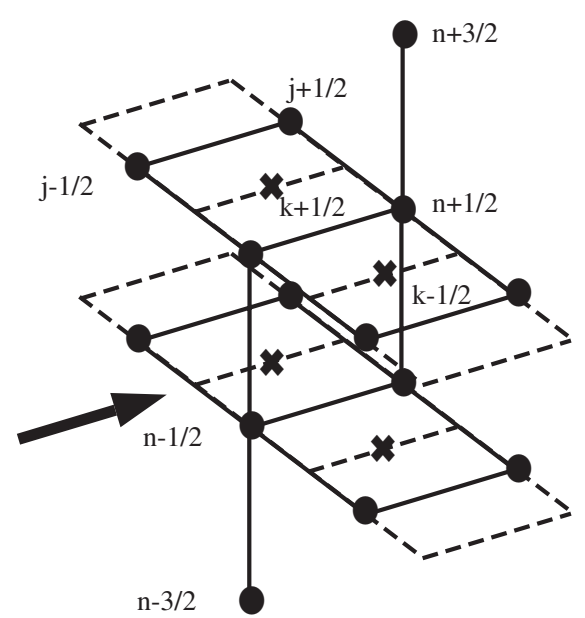

(b)

Figure 11. Four-level second- and fourth-order stencils on staggered grid.

The four-level compact stencil shown in Figure 11(a), is not large enough to eliminate the second-order errors and the smallest stencil on which the error terms could be discretized, is presented in Figure 11(b). With the relation, $\partial u / \partial t=-a \partial u / \partial x-b \partial u / \partial y$, some terms are replaced as below because they could not be discretized on the stencil (Figure 11(b)).

$$
c_{x y y} \Delta x \Delta y^{2} \frac{\partial^{3} u}{\partial x \partial y^{2}}+c_{x x t} \Delta x^{2} \Delta t \frac{\partial^{3} u}{\partial x^{2} \partial t}+c_{x y t} \Delta x \Delta y \Delta t \frac{\partial^{3} u}{\partial x \partial y \partial t}+c_{y y t} \Delta y^{2} \Delta t \frac{\partial^{3} u}{\partial y^{2} \partial t}
$$

where

$$
\begin{aligned}
& c_{x y y}=\frac{v_{y}^{2}-v_{x}^{2}}{12 v_{x}}, \quad c_{x x t}=\frac{\left(10 v_{x}-2\right)(v-1)}{12} \\
& c_{x y t}=\frac{v_{y}\left(10 v_{x}-1\right)\left(2 v_{x}-1\right)}{12 v_{x}}, \quad c_{y y t}=\frac{\left(10 v_{y}^{2}-1\right)}{12}
\end{aligned}
$$

Then the error terms are discretized as

$$
\begin{aligned}
& \Delta x^{2} \Delta t \frac{\partial^{3} u}{\partial x^{2} \partial t}=\left[8 \delta_{t}\left(\mu_{x}-\mu_{y}\right)+\delta_{y}^{2} \delta_{t} \mu_{x}\right] u_{j, k}^{n}, \quad \Delta x \Delta y^{2} \frac{\partial^{3} u}{\partial x \partial y^{2}}=\mu_{t} \delta_{x} \delta_{y}^{2} u_{j, k}^{n} \\
& \Delta y^{2} \Delta t \frac{\partial^{3} u}{\partial y^{2} \partial t}=\delta_{y}^{2} \delta_{t} \mu_{x} u_{j, k}^{n}, \quad \Delta x \Delta y \Delta t \frac{\partial^{3} u}{\partial x \partial y \partial t}=\delta_{t} \mu_{y} \delta_{y} \delta_{x} u_{j, k}^{n}
\end{aligned}
$$




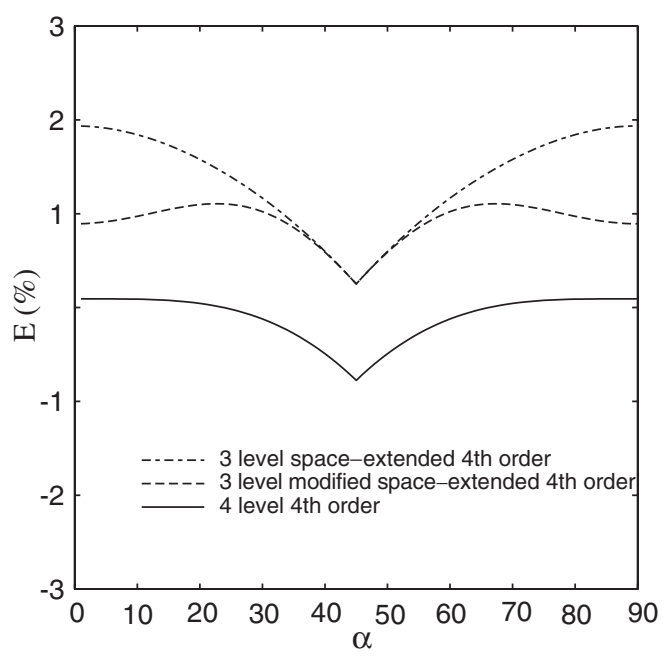

Figure 12. Comparison of three fourth-order upwind leapfrog methods for advection problems. Grid points per wavelength, $N=4$. Courant number, $v=1 / 4$.

and leading to the four-level fourth-order upwind leapfrog method

$$
\begin{aligned}
& \delta_{t} u_{j+1 / 2, k}^{n+1}+\delta_{t} u_{j-1 / 2, k}^{n-1}+2\left(v_{x} \delta_{x}+v_{y} \delta_{y}\right) \mu_{t} u_{j, k}^{n} \\
& \quad-\left\{c_{x y y} \mu_{t} \delta_{x} \delta_{y}^{2}+c_{x x t}\left[8 \delta_{t}\left(\mu_{x}-\mu_{y}\right)+\delta_{y}^{2} \delta_{t} \mu_{x}\right]+c_{x y t} \delta_{t} \mu_{y} \delta_{y} \delta_{x}+c_{y y t} \delta_{y}^{2} \delta_{t} \mu_{x}\right\} u_{j, k}^{n}=0
\end{aligned}
$$

Figure 12 shows the errors of three different fourth-order schemes when the number of cellsper-wavelength, $N=4$ and the wave front angle is parallel to the propagation direction as explained in the Section 3.2.

The two three-level schemes contain small errors when the solution is advected oblique to the co-ordinates, but the modified scheme with a more compact stencil has an error around one-half that of the basic space-extended scheme. The modified scheme compared with the basic one, has the property that its stencil is more compact, which can reduce the boundary problem, and it has higher resolution. The four-level scheme has a more compact stencil and better resolution than the three-level ones but its maximum Courant number is half of theirs.

\subsection{Numerical experiment}

Three test problems are presented to compare the three- and four-level upwind leapfrog schemes based on Figures 10 and 11. An initial distribution is advected along a straight line, circle or given local velocity. The explanation of the rotating disk simulation is presented in Figure 13(a). This example rotates the initial distribution around the origin of the co-ordinates as though it were a solid body. Figure 13(b) explains the Stokes flow advection. A Gaussian distribution is advected along the Stokes flow speed. 


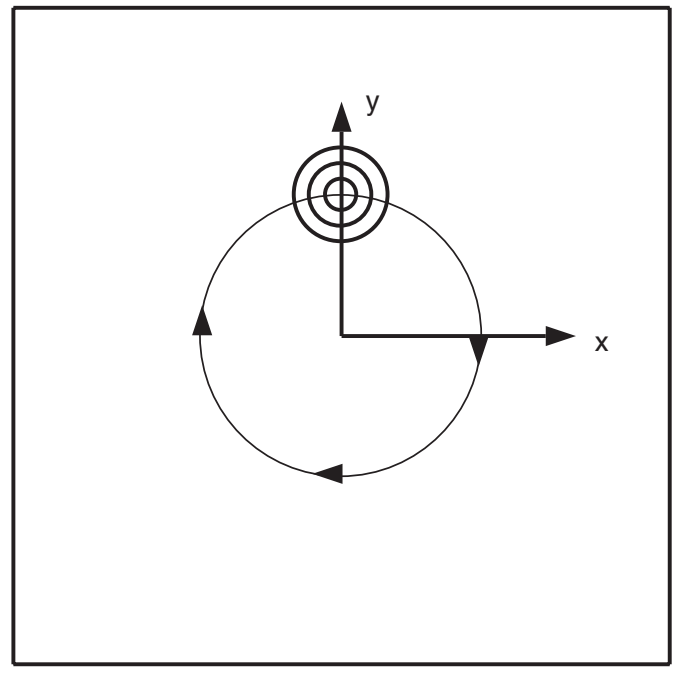

(a)

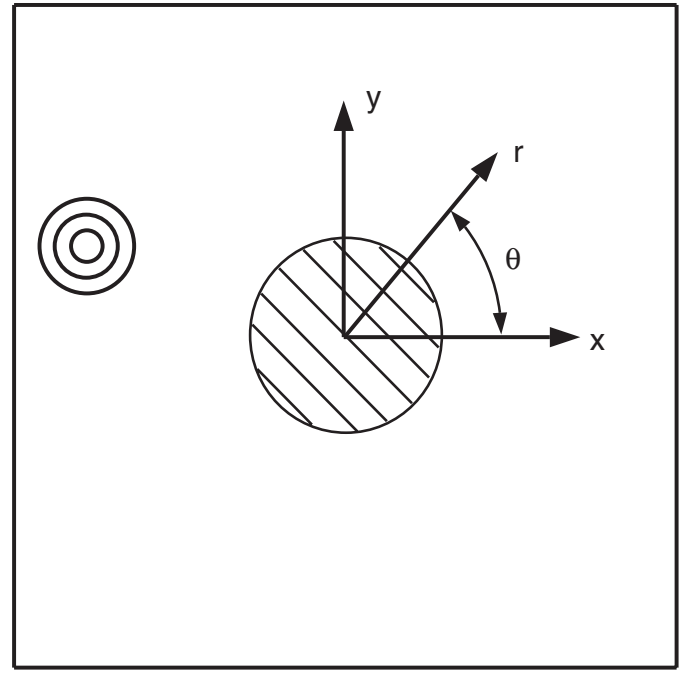

(b)

Figure 13. Illustration of test cases for flow advection simulation: (a) rotating disk advection; and (b) stokes flow advection.

3.4.1. Simple advection problem. An initial distribution as described below is advected along one direction at constant speed. $\S$

$$
u(x, y)=\exp \left[-\frac{\ln 2}{0.2^{2}}\left(x^{2}+y^{2}\right)\right]
$$

The computational domain is $-2 \leqslant x \leqslant 2,-2 \leqslant y \leqslant 2$, the grid size is $\Delta x=\Delta y=0.1$ and time step is $\Delta t=0.04$. We stress that this distribution is very underresolved on the mesh and 5000 iterations were performed. The initial distribution is revolved through the computational domain 50 times. Figure 14(a)-14(c) show the results updated by the three-level scheme. The result advected along $x$-axis, appears in Figure 14(a). It shows lagging errors and its peak value is decreased to $85.1 \%$ of the initial profile. Figure $14(\mathrm{~b})$ presents the result advected at the velocity of $(a=1.0, b=0.5)$ and having lagging errors normal to the propagation direction. Their peak value is just $74 \%$ of the initial one. The distribution advected along the diagonal line $\left(\alpha=45^{\circ}\right)$ appears in Figure 14(c) and shows slight leading phase errors. The advected results with the four-level fourth-order schemes appear in Figure 14(d)-14(f). When the distribution is advected along the $x$-axis, it preserves the initial profile very accurately and its peak value is $98.2 \%$ of its initial peak. Figure 14(e) presents the result advected at the speed, $a=1.0, b=0.5$. Although it is mainly advected along $x$-axis, it does not show any significant error along $x$-axis but reveals large leading errors along $y$-axis. Its peak value is decreased to $80.8 \%$ of the exact one. When the distribution is advected along the diagonal

\footnotetext{
$\S$ It is generally agreed now that advection in a constant direction can be a more strict test of a scheme than the formerly common test of advection in a circular path. This is because in the latter test there may be some cancellation of the errors incurred by moving in all the different directions.
} 

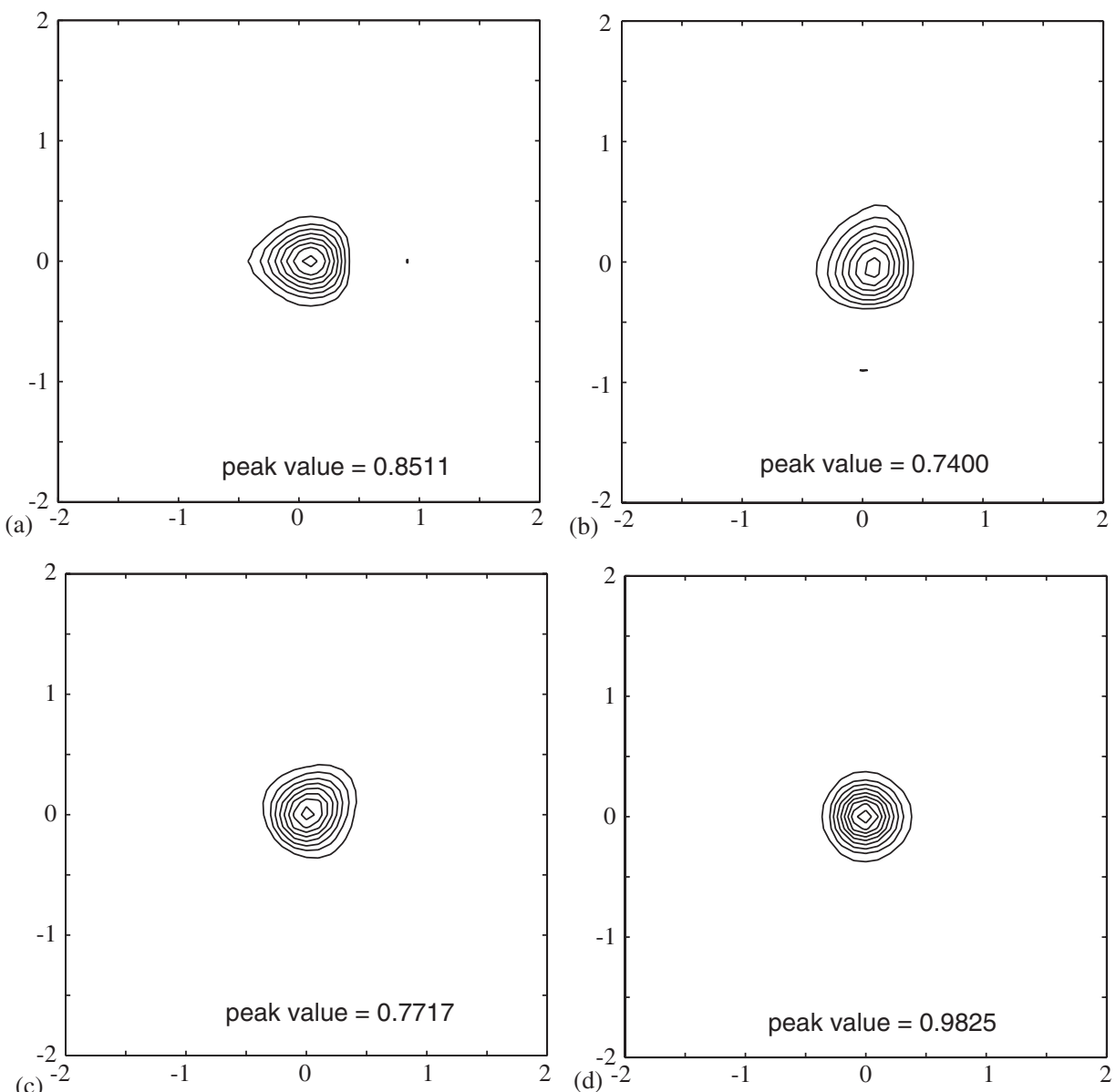

(c)
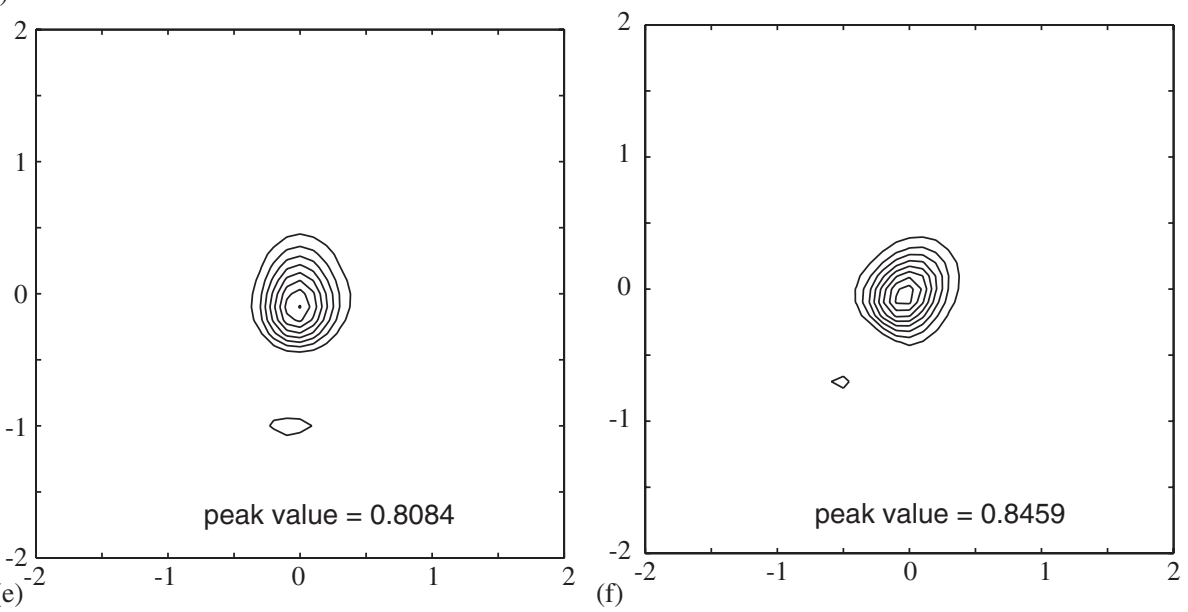

Figure 14. Numerical results of three- and four-level fourth-order upwind leapfrog methods. (a), (b), (c): Three-level, (d), (e), (f): Four-level. $\Delta x=\Delta y=0.1 . \Delta t=0.04, t=200.0$. Contour level : 0.1-1.0. (a) $a=1, b=0$; (b) $a=1, b=0.5$; (c) $a=1, b=1$; (d) $a=1, b=0$; (e) $a=1, b=0.5$; and (f) $a=1, b=1$. 
line, the result reveals leading errors along the propagation direction. The maximum value is $84.1 \%$ of the exact value.

3.4.2. Rotating disk. We also consider the case of advection in a circle, governed by the equation

$$
\frac{\partial u}{\partial t}+y \frac{\partial u}{\partial x}-x \frac{\partial u}{\partial y}=0
$$

Figure 15 presents the results of simulating the above Equation (22) with the four-level fourth-order scheme on a $20 \times 20$ grid. Although this is in some ways a more benign test than advection along a straight path, for reasons explained above (see Section 2.3), it is necessary to include additional terms in the scheme to account for the non-constant propagation velocity. If this is not done, the scheme is merely second-order accurate, and after one revolution $(t=\pi)$, the distribution starts to distort, Figure 15(a). The solution does not preserve the initial shape after one revolution, Figure 15(b) and the measured peak values are meaningless, Figure 15(b,c).

To improve the solution some additional terms, derived by expanding the second-order truncation terms and differentiating the analytic wave speed, $\mathbf{a}=(y,-x)$ are discretized and subtracted from the four-level scheme (20). To achieve the fourth-order accuracy, additional truncation error terms should be discretized and substracted from the four-level scheme (20) as explained in the Section 2.3. However it is not possible to discretize the error terms in conservative form on the four-level stencil shown in Figure 11(b) and the advection speed is smooth over the computational domain, the truncation error terms are expanded at the centre of the stencil and simplified by substituting the analytic advection speed as follows:

$$
-\left(c_{x x} \delta_{x}^{2}+c_{x y} \delta_{x} \delta_{y}+c_{y y} \delta_{y}^{2}+c_{x t} \delta_{x} \delta_{t}+c_{y t} \delta_{y} \delta_{t}\right) u_{j, k}^{n}
$$

where

$$
\begin{aligned}
& c_{x x}=-\frac{\bar{v}_{y}}{12 \bar{v}_{x}} \delta_{y} \bar{v}_{x}, \quad c_{x y}=\frac{1}{6}\left(\delta_{y} \bar{v}_{x}+\delta_{x} \bar{v}_{y}\right), \quad c_{y y}=-\frac{\bar{v}_{y}}{12 \bar{v}_{x}} \delta_{x} \bar{v}_{y} \\
& c_{x t}=-\frac{5}{12} \bar{v}_{y} \delta_{y} \bar{v}_{x}, \quad c_{y t}=\frac{1}{12}\left(6-5 \bar{v}_{x}\right) \delta_{x} \bar{v}_{y}
\end{aligned}
$$

and $\bar{v}_{x}=\left.v_{x}\right|_{j, k}, \bar{v}_{y}=\left.v_{y}\right|_{j, k}$. Those terms are discretized and subtracted from the four-level scheme (20). This correction improves the scheme and leads to the results shown in Figure 15(d)-15(f). The modified scheme did not change the initial distribution and generate any disturbance. Even four revolution did not distort the initial distribution and its peak value decreases steadily by $5.75 \%$ over the course of four revolution. The above correction term (23) is implemented for the scalar advection mainly transported along $+x$ direction and corrections for other direction are presented in Appendix A.

3.4.3. Stokes flow advection. In this section, we compose a problem that is a little more representative of practical difficulties. We use Stokes flow around a cylinder to typify the kind of flow that would greatly stretch and distort any initial distribution. Therefore, the 

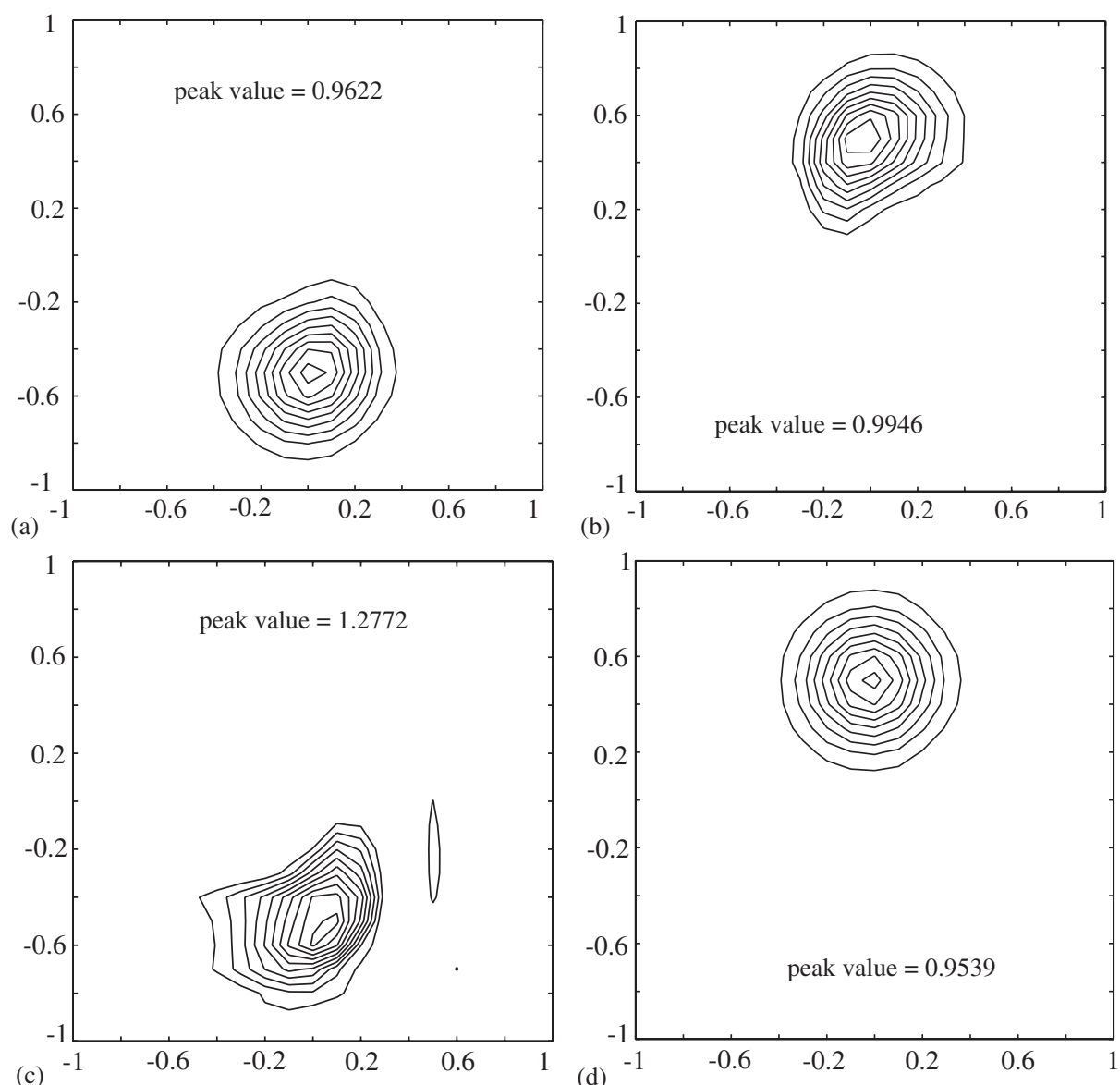

(b)
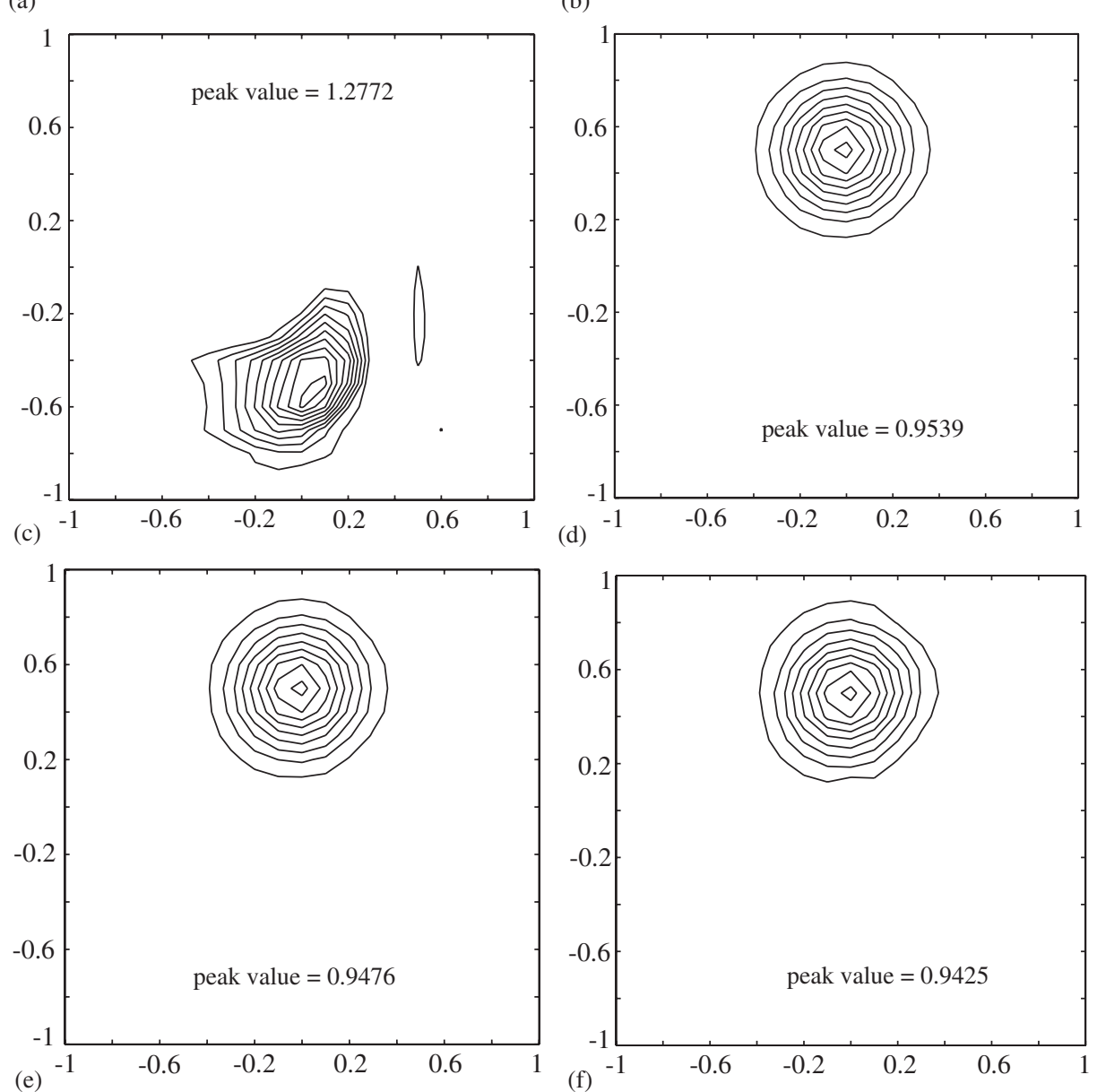

Figure 15. Rotating disk simulation with second- and fourth-order upwind leapfrog methods. Grid size $=20 \times 20$. (a), (b), (c): second order, (d), (e), (f): fourth order. (a) $t=\pi$; (b) $t=2 \pi$; (c) $t=3 \pi$; (d) $t=2 \pi$; (e) $t=4 \pi$; and (f) $t=8 \pi$. 
governing equation is

$$
\frac{\partial u}{\partial t}+a_{r} \frac{\partial u}{\partial r}+a_{\theta} \frac{1}{r} \frac{\partial u}{\partial \theta}=0
$$

where

$$
\begin{aligned}
& a_{r}=U_{\infty} \cos \left(1+\frac{R^{3}}{2 r^{3}}-\frac{3 R}{2 r}\right) \\
& a_{\theta}=U_{\infty} \sin \left(-1+\frac{R^{3}}{4 r^{3}}+\frac{3 R}{4 r}\right)
\end{aligned}
$$

The uniform velocity, $U_{\infty}$, and cylinder radius, $R$, are given the value 1.0. To implement the upwind leapfrog schemes developed on a uniform spacing grid, it is necessary to transform the governing equation to the computational domain. It is then simply solved by changing the advection velocity. The governing equation in the computational domain is

$$
\frac{\partial u}{\partial t}+a_{\xi} \frac{\partial u}{\partial \xi}+a_{\eta} \frac{\partial u}{\partial \eta}=0
$$

where $a_{\xi}=a_{r} \partial \xi / \partial r$ and $a_{\eta}=a_{\theta} / r \partial \eta / \partial \theta$.

Again a Gaussian distribution is located at $(-4,1)$ relative to the centre of the cylinder, i.e.

$$
u(x, y)=\exp \left\{-\frac{\ln 2}{0.3^{2}}\left[(x+4)^{2}+(y-1)^{2}\right]\right\}
$$

and advected along the local advection speed, $\left(a_{\xi}, a_{\eta}\right)$ which requires special treatment for the truncation error terms as discussed in Section 2.3. The truncation error terms for the second-order scheme are again presented as

$$
\begin{aligned}
& \delta_{t} u_{j+1 / 2, k}^{n+1 / 2}+\delta_{t} u_{j-1 / 2, k}^{n-1 / 2}+2\left(v_{x} \delta_{x} u_{j, k}^{n}+v_{y} \delta_{y} u_{j, k}^{n}\right)=2 \Delta t\left(\frac{\partial u}{\partial t}+a \frac{\partial u}{\partial x}+b \frac{\partial u}{\partial y}\right) \\
& +\frac{\Delta t \Delta x^{2}}{12}\left[a \frac{\partial^{3} u}{\partial x^{3}}+b \frac{\Delta y^{2}}{\Delta x^{2}} \frac{\partial^{3} u}{\partial y^{3}}+3 \frac{\partial^{3} u}{\partial x^{2} \partial t}+6 \frac{\Delta t}{\Delta x} \frac{\partial^{3} u}{\partial x \partial t^{2}}+4 \frac{\Delta t^{2}}{\Delta x^{2}} \frac{\partial^{3} u}{\partial t^{3}}\right]+O\left(\Delta x^{4}\right)
\end{aligned}
$$

The relation $\partial u / \partial t=-a \partial u / \partial x-b \partial u / \partial y$, simplifies the error terms as

$$
\begin{aligned}
& a \frac{\partial^{3} u}{\partial x^{3}}+b \frac{\Delta y^{2}}{\Delta x^{2}} \frac{\partial^{3} u}{\partial y^{3}}-3 \frac{\partial^{2}}{\partial x^{2}}\left(a \frac{\partial u}{\partial x}+b \frac{\partial u}{\partial y}\right) \\
& +\left[\left(6 \frac{\Delta t}{\Delta x}-4 a \frac{\Delta t^{2}}{\Delta x^{2}}\right) \frac{\partial}{\partial x}-4 b \frac{\Delta t^{2}}{\Delta x^{2}} \frac{\partial}{\partial y}\right] \frac{\partial^{2} u}{\partial t^{2}}
\end{aligned}
$$




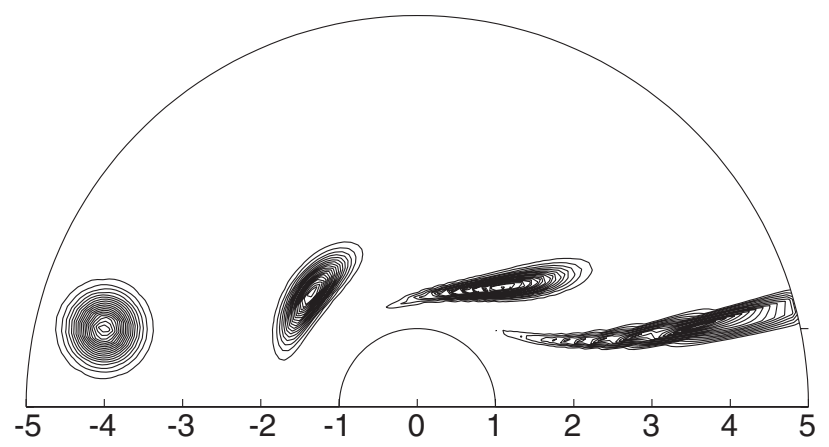

Figure 16. Result of Stokes flow advection. Grid size $=60 \times 90$.

and leads to $\partial^{2} u / \partial t^{2}=(a \partial / \partial x+b \partial / \partial y)^{2} u$. The above terms are discretized on the stencil (Figure 5(a)) and the resultant scheme implemented for the computation is

$$
\begin{aligned}
& \delta_{t} u_{j+1 / 2, k}^{n+1 / 2}+\delta_{t} u_{j-1 / 2, k}^{n-1 / 2}+2\left(\bar{v}_{x} \delta_{x}+\bar{v}_{y} \delta_{y}\right) u_{j, k}^{n}-\frac{1}{12}\left\{\bar{v}_{x} \delta_{x}^{3}+\bar{v}_{y} \delta_{y}^{3}\right. \\
& \left.\quad-3 \delta_{x}^{2}\left(\bar{v}_{x} \delta_{x}+\bar{v}_{y} \delta_{y}\right)-\left[\left(4 \bar{v}_{x}-6\right) \delta_{x}-4 \bar{v}_{y} \delta_{y}\right]\left(\bar{v}_{x} \delta_{x}+\bar{v}_{y} \delta_{y}\right)^{2}\right\} u_{j, k}^{n}=0
\end{aligned}
$$

The computational domain is defined as $1 \leqslant r \leqslant 5$ and $0 \leqslant \theta \leqslant \pi$. The grid size is $60 \times 90$ and the computation time is $0 \leqslant t \leqslant 15$. As the Gaussian distribution passes around the cylinder, a small part of it, which passes closest to the surface, is severely retarded, while other part moves more freely. Therefore, the outcome is a drastic distortion of the profile. The initial distribution is advected at a different velocity and stretched severely, as shown in Figure 16. At early time $(t=5.0)$, the result still preserved the initial profile but it is stretched very much $(t=10.0)$. Later the resulting profile did not resemble the initial profile any more $(t=15.0)$. Further development of these advection schemes may be of interest in its own right, for example in the calculation of pollutant dispersal or meteorological flows. In the aeroacoustic context these schemes are needed to predict advected quantities such as entropy or vorticity.

\section{CONCLUSION}

In the present work, a 1D version of the upwind leapfrog method was successfully extended to multi-dimensional advection problems. This work is an attempt at creating highly accurate schemes for passive advection of scalar quantity. By design, these methods are free from dissipation and the dispersion error could be reduced significantly by keeping the stencil compact in the characteristic co-ordinate. The fourth-order methods are developed with full accuracy retained up to boundaries and numerical experiment results of several test cases also demonstrated the accuracy of the upwind leapfrog method. Application of this method to various examples is strongly recommended to further develop these techniques. 


\section{APPENDIX A}

The sets of the upwind leapfrog methods and correction terms for all direction are described below. They are three- and four-level fourth-order methods and correction terms for rotating disk and Stokes flow simulation.

\section{A.1. Set of three-level fourth-order schemes}

$+x$ axis:

$$
\delta_{t} u_{j+1 / 2, k}^{n+1 / 2}+\delta_{t} u_{j-1 / 2, k}^{n-1 / 2}+2\left(v_{x} \delta_{x}+v_{y} \delta_{y}\right) u_{j, k}^{n}+\left(c_{x x x} \delta_{x}^{3}+c_{x x y} \delta_{x}^{2} \delta_{y}+c_{x y y} \delta_{x} \delta_{y}^{2}+c_{y y y} \delta_{y}^{3}\right) u_{j, k}^{n}=0
$$

$-x$ axis:

$$
\delta_{t} u_{j-1 / 2, k}^{n+1 / 2}+\delta_{t} u_{j+1 / 2, k}^{n-1 / 2}+2\left(v_{x} \delta_{x}+v_{y} \delta_{y}\right) u_{j, k}^{n}+\left(c_{x x x} \delta_{x}^{3}+c_{x x y} \delta_{x}^{2} \delta_{y}+c_{x y y} \delta_{x} \delta_{y}^{2}+c_{y y y} \delta_{y}^{3}\right) u_{j, k}^{n}=0
$$

$+y$ axis:

$$
\delta_{t} u_{j, k+1 / 2}^{n+1 / 2}+\delta_{t} u_{j, k-1 / 2}^{n-1 / 2}+2\left(v_{x} \delta_{x}+v_{y} \delta_{y}\right) u_{j, k}^{n}+\left(c_{x x x} \delta_{x}^{3}+c_{x x y} \delta_{x}^{2} \delta_{y}+c_{x y y} \delta_{x} \delta_{y}^{2}+c_{y y y} \delta_{y}^{3}\right) u_{j, k}^{n}=0
$$

$-y$ axis:

$$
\delta_{t} u_{j, k-1 / 2}^{n+1 / 2}+\delta_{t} u_{j, k+1 / 2}^{n-1 / 2}+2\left(v_{x} \delta_{x}+v_{y} \delta_{y}\right) u_{j, k}^{n}+\left(c_{x x x} \delta_{x}^{3}+c_{x x y} \delta_{x}^{2} \delta_{y}+c_{x y y} \delta_{x} \delta_{y}^{2}+c_{y y y} \delta_{y}^{3}\right) u_{j, k}^{n}=0
$$

where

\begin{tabular}{|l|l|l|l|l|}
\hline & $12 \times c_{x x x}$ & $4 \times c_{x x y}$ & $4 \times c_{x y y}$ & $12 \times c_{y y y}$ \\
\hline$+x$ axis & $2 v_{x}\left(2 v_{x}^{2}-3 v_{x}+1\right)$ & $v_{y}\left(2 v_{x}-1\right)^{2}$ & $2 v_{y}^{2}\left(2 v_{x}-1\right)$ & $v_{y}\left(4 v_{y}^{2}-1\right)$ \\
\hline$-x$ axis & $2 v_{x}\left(2 v_{x}^{2}+3 v_{x}+1\right)$ & $v_{y}\left(2 v_{x}+1\right)^{2}$ & $2 v_{y}^{2}\left(2 v_{x}+1\right)$ & $v_{y}\left(4 v_{y}^{2}-1\right)$ \\
\hline$+y$ axis & $v_{x}\left(4 v_{x}^{2}-1\right)$ & $2 v_{x}^{2}\left(2 v_{y}-1\right)$ & $v_{x}\left(2 v_{y}-1\right)^{2}$ & $2 v_{y}\left(2 v_{y}^{2}-3 v_{y}+1\right)$ \\
\hline$-y$ axis & $v_{x}\left(4 v_{x}^{2}-1\right)$ & $2 v_{x}^{2}\left(2 v_{y}+1\right)$ & $v_{x}\left(2 v_{y}+1\right)^{2}$ & $2 v_{y}\left(2 v_{y}^{2}+3 v_{y}+1\right)$ \\
\hline
\end{tabular}

\section{A.2. Set of four-level fourth-order schemes}

$$
\begin{aligned}
& +x \text { axis: } \delta_{t} u_{j+1 / 2, k}^{n+1}+\delta_{t} u_{j-1 / 2, k}^{n-1}+2\left(v_{x} \delta_{x}+v_{y} \delta_{y}\right) \mu_{t} u_{j, k}^{n} \\
& \quad-\left\{c_{x y y} \mu_{t} \delta_{x} \delta_{y}^{2}+c_{x x t}\left[8 \delta_{t}\left(\mu_{x}-\mu_{y}\right)+\delta_{y}^{2} \delta_{t} \mu_{x}\right]+c_{x y t} \delta_{t} \mu_{y} \delta_{y} \delta_{x}+c_{y y t} \delta_{y}^{2} \delta_{t} \mu_{x}\right\} u_{j, k}^{n}=0 \\
& -x \text { axis: } \delta_{t} u_{j-1 / 2, k}^{n+1}+\delta_{t} u_{j+1 / 2, k}^{n-1}+2\left(v_{x} \delta_{x}+v_{y} \delta_{y}\right) \mu_{t} u_{j, k}^{n} \\
& \quad-\left\{c_{x y y} \mu_{t} \delta_{x} \delta_{y}^{2}+c_{x x t}\left[8 \delta_{t}\left(\mu_{x}-\mu_{y}\right)+\delta_{y}^{2} \delta_{t} \mu_{x}\right]+c_{x y t} \delta_{t} \mu_{y} \delta_{y} \delta_{x}+c_{y y t} \delta_{y}^{2} \delta_{t} \mu_{x}\right\} u_{j, k}^{n}=0 \\
& +y \text { axis: } \delta_{t} u_{j, k+1 / 2}^{n+1}+\delta_{t} u_{j, k-1 / 2}^{n-1}+2\left(v_{x} \delta_{x}+v_{y} \delta_{y}\right) \mu_{t} u_{j, k}^{n}
\end{aligned}
$$




$$
\begin{aligned}
& -\left\{c_{x x y} \mu_{t} \delta_{x}^{2} \delta_{y}+c_{y y t}\left[8 \delta_{t}\left(\mu_{y}-\mu_{x}\right)+\delta_{x}^{2} \delta_{t} \mu_{y}\right]+c_{x y t} \delta_{t} \mu_{x} \delta_{x} \delta_{y}+c_{x x t} \delta_{x}^{2} \delta_{t} \mu_{y}\right\} u_{j, k}^{n}=0 \\
& -y \text { axis: } \delta_{t} u_{j, k-1 / 2}^{n+1}+\delta_{t} u_{j, k+1 / 2}^{n-1}+2\left(v_{x} \delta_{x}+v_{y} \delta_{y}\right) \mu_{t} u_{j, k}^{n} \\
& -\left\{c_{x x y} \mu_{t} \delta_{x}^{2} \delta_{y}+c_{y y t}\left[8 \delta_{t}\left(\mu_{y}-\mu_{x}\right)+\delta_{x}^{2} \delta_{t} \mu_{y}\right]+c_{x y t} \delta_{t} \mu_{x} \delta_{x} \delta_{y}+c_{x x t} \delta_{x}^{2} \delta_{t} \mu_{y}\right\} u_{j, k}^{n}=0
\end{aligned}
$$

where

\begin{tabular}{|l|l|l|l|l|}
\hline & $12 \times c_{x y y}$ & $12 \times c_{x x t}$ & $12 \times c_{x y t}$ & $12 \times c_{y y t}$ \\
\hline$+x$ axis & $\left(v_{y}^{2}-v_{x}^{2}\right) / v_{x}$ & $10 v_{x}^{2}-12 v_{x}+2$ & $\frac{v_{y}}{v_{x}}\left(20 v_{x}^{2}-12 v_{x}+1\right)$ & $10 v_{y}^{2}-1$ \\
\hline$-x$ axis & $\left(v_{y}^{2}-v_{x}^{2}\right) / v_{x}$ & $10 v_{x}^{2}+12 v_{x}+2$ & $\frac{v_{y}}{v_{x}}\left(20 v_{x}^{2}+12 v_{x}+1\right)$ & $10 v_{y}^{2}-1$ \\
\hline $12 \times c_{x x y}$ & $12 \times c_{x x t}$ & $12 \times c_{x y t}$ & $12 \times c_{y y t}$ \\
\hline$+y$ axis & $\left(v_{x}^{2}-v_{y}^{2}\right) / v_{y}$ & $10 v_{x}^{2}-1$ & $\frac{v_{x}}{v_{y}}\left(20 v_{y}^{2}-12 v_{y}+1\right)$ & $10 v_{y}^{2}-12 v_{y}+2$ \\
\hline$-y$ axis & $\left(v_{x}^{2}-v_{y}^{2}\right) / v_{y}$ & $10 v_{x}^{2}-1$ & $\frac{v_{x}}{v_{y}}\left(20 v_{y}^{2}+12 v_{y}+1\right)$ & $10 v_{y}^{2}+12 v_{y}+2$ \\
\hline
\end{tabular}

\section{A.3. Set of fourth-order schemes for rotating disk simulation}

\begin{tabular}{|l|l|l|l|l|l|}
\hline & $12 \times c_{x x}$ & $12 \times c_{x y}$ & $12 \times c_{y y}$ & $12 \times c_{x t}$ & $12 \times c_{y t}$ \\
\hline$+x$ axis & $-\left(\frac{\bar{v}_{y}}{\bar{v}_{x}}\right) \delta_{y} \bar{v}_{x}$ & $2\left(\delta_{y} \bar{v}_{x}+\delta_{x} \bar{v}_{y}\right)$ & $-\left(\frac{\bar{v}_{y}}{\bar{v}_{x}}\right) \delta_{x} \bar{v}_{y}$ & $-5 \bar{v}_{y} \delta_{y} \bar{v}_{x}$ & $\left(6-5 \bar{v}_{x}\right) \delta_{x} \bar{v}_{y}$ \\
\hline$-x$ axis & $-\left(\frac{\bar{v}_{y}}{\bar{v}_{x}}\right) \delta_{y} \bar{v}_{x}$ & $2\left(\delta_{y} \bar{v}_{x}+\delta_{x} \bar{v}_{y}\right)$ & $-\left(\frac{\bar{v}_{y}}{\bar{v}_{x}}\right) \delta_{x} \bar{v}_{y}$ & $-5 \bar{v}_{y} \delta_{y} \bar{v}_{x}$ & $-\left(6+5 \bar{v}_{x}\right) \delta_{x} \bar{v}_{y}$ \\
\hline$+y$ axis & $-\left(\frac{\bar{v}_{x}}{\bar{v}_{y}}\right) \delta_{y} \bar{v}_{x}$ & $2\left(\delta_{y} \bar{v}_{x}+\delta_{x} \bar{v}_{y}\right)$ & $-\left(\frac{\bar{v}_{x}}{\bar{v}_{y}}\right) \delta_{x} \bar{v}_{y}$ & $\left(6-5 \bar{v}_{y}\right) \delta_{y} \bar{v}_{x}$ & $-5 \bar{v}_{x} \delta_{x} \bar{v}_{y}$ \\
\hline$-y$ axis & $-\left(\frac{\bar{v}_{x}}{\bar{v}_{y}}\right) \delta_{y} \bar{v}_{x}$ & $2\left(\delta_{y} \bar{v}_{x}+\delta_{x} \bar{v}_{y}\right)$ & $-\left(\frac{\bar{v}_{x}}{\bar{v}_{y}}\right) \delta_{x} \bar{v}_{y}$ & $-\left(6+5 \bar{v}_{y}\right) \delta_{y} \bar{v}_{x}$ & $-5 \bar{v}_{x} \delta_{x} \bar{v}_{y}$ \\
\hline
\end{tabular}

A.4. Set of fourth-order schemes for stokes flow simulation

$$
\begin{aligned}
& +x \text { axis: } \delta_{t} u_{j+1 / 2, k}^{n+1 / 2}+\delta_{t} u_{j-1 / 2, k}^{n-1 / 2}+2\left(\bar{v}_{x} \delta_{x}+\bar{v}_{y} \delta_{y}\right) u_{j, k}^{n} \\
& \quad-\frac{1}{12}\left\{\bar{v}_{x} \delta_{x}^{3}+\bar{v}_{y} \delta_{y}^{3}-3 \delta_{x}^{2}\left(\bar{v}_{x} \delta_{x}+\bar{v}_{y} \delta_{y}\right)-\left[\left(4 \bar{v}_{x}-6\right) \delta_{x}-4 \bar{v}_{y} \delta_{y}\right]\left(\bar{v}_{x} \delta_{x}+\bar{v}_{y} \delta_{y}\right)^{2}\right\} u_{j, k}^{n}=0
\end{aligned}
$$




$$
\begin{aligned}
& -x \text { axis: } \delta_{t} u_{j+1 / 2, k}^{n+1 / 2}+\delta_{t} u_{j-1 / 2, k}^{n-1 / 2}+2\left(\bar{v}_{x} \delta_{x}+\bar{v}_{y} \delta_{y}\right) u_{j, k}^{n} \\
& -\frac{1}{12}\left\{\bar{v}_{x} \delta_{x}^{3}+\bar{v}_{y} \delta_{y}^{3}-3 \delta_{x}^{2}\left(\bar{v}_{x} \delta_{x}+\bar{v}_{y} \delta_{y}\right)-\left[\left(4 \bar{v}_{x}+6\right) \delta_{x}-4 \bar{v}_{y} \delta_{y}\right]\left(\bar{v}_{x} \delta_{x}+\bar{v}_{y} \delta_{y}\right)^{2}\right\} u_{j, k}^{n}=0 \\
& +y \text { axis: } \delta_{t} u_{j, k+1 / 2}^{n+1 / 2}+\delta_{t} u_{j, k-1 / 2}^{n-1 / 2}+2\left(\bar{v}_{x} \delta_{x}+\bar{v}_{y} \delta_{y}\right) u_{j, k}^{n} \\
& \quad-\frac{1}{12}\left\{\bar{v}_{x} \delta_{x}^{3}+\bar{v}_{y} \delta_{y}^{3}-3 \delta_{y}^{2}\left(\bar{v}_{x} \delta_{x}+\bar{v}_{y} \delta_{y}\right)-\left[\left(4 \bar{v}_{y}-6\right) \delta_{y}-4 \bar{v}_{x} \delta_{x}\right]\left(\bar{v}_{x} \delta_{x}+\bar{v}_{y} \delta_{y}\right)^{2}\right\} u_{j, k}^{n}=0 \\
& -y \text { axis: } \delta_{t} u_{j, k+1 / 2}^{n+1 / 2}+\delta_{t} u_{j, k-1 / 2}^{n-1 / 2}+2\left(\bar{v}_{x} \delta_{x}+\bar{v}_{y} \delta_{y}\right) u_{j, k}^{n} \\
& \quad-\frac{1}{12}\left\{\bar{v}_{x} \delta_{x}^{3}+\bar{v}_{y} \delta_{y}^{3}-3 \delta_{y}^{2}\left(\bar{v}_{x} \delta_{x}+\bar{v}_{y} \delta_{y}\right)-\left[\left(4 \bar{v}_{y}+6\right) \delta_{y}-4 \bar{v}_{x} \delta_{x}\right]\left(\bar{v}_{x} \delta_{x}+\bar{v}_{y} \delta_{y}\right)^{2}\right\} u_{j, k}^{n}=0
\end{aligned}
$$

\section{ACKNOWLEDGEMENTS}

I would like to express my deep appreciation to Prof. P. L. Roe for his insight and thoughtful discussion on this research.

\section{REFERENCES}

1. Iserles A. Generalised leapfrog methods. IMA Journal of Numerical Analysis 1986; 6.

2. Roe PL. Linear bicharacteristic schemes without dissipation. SIAM Journal on Scientific Computing 1998; 19(5):1405-1427.

3. Thomas JP, Roe PL. Development of non-dissipative numerical schemes for computational aeroacoustics. AIAA Paper 93-3382, AIAA 11th Computational Fluid Dynamics Conference, 1993.

4. Thomas JP, Kim C, Roe PL. Progress towards a new computational scheme for aeroacoustics. AIAA Paper 95-1758, AIAA 12th Computational Fluid Dynamics Conference, 1995.

5. Thomas JP. Ph.D. Thesis, Department of Aerospace Engineering, University of Michigan, 1996. 